$$
\text { NYSERDA-- 960/1910 }
$$

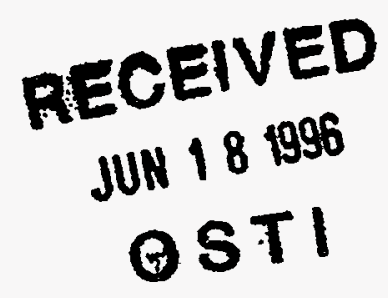

PATTERNS AND TRENDS

New York State Energy Profiles: 1980-1994

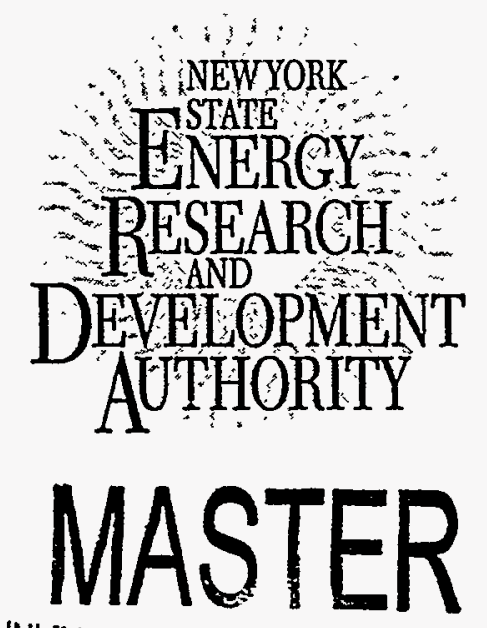


The New York State Energy Research and Development Authority (NYSERDA) is a public benefit corporation created in 1975 by the New York State Legislature. F. William Valentino is President and Chief Operating Officer.

NYSERDA's primary mission is to carry out a broad program of energy research, development and demonstration projects designed to develop and apply efficient technologies to help ensure that New York has secure and economical future supplies of energy, while protecting environmental values and promoting economic growth.

NYSERDA derives its basic research revenues from an assessment levied on the intrastate sales of New York State's investor-owned electric and gas utilities. Additional research dollars come from limited corporate funds and a voluntary annual contribution by the New York Power Authority.

In its research program, NYSERDA stresses consultation and collaboration with other organizations, including utilities, universities, industries, private engineering and scientific research firms, local governments, and State and federal agencies. These efforts stretch NYSERDA's limited research funds and ensure the involvement of those who can use the results of the research.

In its federally funded Energy Services program, NYSERDA provides technical assistance to improve the energy and environmental performance of businesses and institutions, helps secure energy-project funding from private and public sources, and converts fleet vehicles to alternative fuels. The Energy Analysis program focuses on using energy, regulatory, and environmental policies to help New York State businesses grow and to meet the needs of New York State's energy consumers.

NYSERDA also has responsibility for:

- Managing the 3,300-acre Western New York Nuclear Service Center at West Valley 35 miles south of Buffalo, the site of a former commercial nuclear fuel reprocessing plant and a low-level radioactive waste disposal area. These responsibilities include:

- Participating in the West Valley Demonstration Project, a joint federal/State effort to solidify the high-level radioactive wastes left over from the reprocessing operation and to clean up the facilities used.

- Maintaining the portion of the site not being used in the Demonstration Project, including the shut-down low-level radioactive waste disposal area.

- Issuing tax-exempt bonds to finance facilities for electric and gas utilities and energy projects for private companies.

- Constructing and operating facilities for disposal of low-level radioactive wastes produced in New York State, once the State makes disposal method and site decisions and approvals have been issued by State regulatory agencies.

- Managing a 365-acre portion of a Superfund clean-up site in Malta, 20 miles north of Albany. Part of the site was once owned by the federal government. Portions of it have been used by the federal government and its contractors since the 1940s for activities that have included rocket engine and fuel testing, weapons testing, and space research.

For more information, contact the Technical Communications unit, NYSERDA, 2 Empire State Plaza, Suite 1901, Albany, New York 12223-1253, (518) 465-6251, ext. 250.

State of New York George E. Pataki, Governor
Energy Research and Development Authority

F. William Valentino, President 


\section{MESSAGE FROM THE PRESIDENT}

New York State Energy Profiles: 1980-1994 is the first annual comprehensive energy overview published by the New York State Energy Research and Development Authority (NYSERDA). It is intended to provide energy analysts, policymakers, and consumers with information about energy-use trends in New York State.

This publication documents basic energy consumption, sources of supply, and price and expenditure data for New York State.

In attempting to present the data in a manner readily understandable to a broad audience, we have used summaries, graphs, and tables not only in physical units, such as barrels and tons, but also in British thermal units to allow easier comparisons among fuels.

One of NYSERDA's missions is to help the citizens of New York State make sound decisions for a secure, affordable, and environmentally acceptable energy future. We hope New York State Energy Profiles will help further that goal.

F. William Valentino

President

New York State Energy Research and Development Authority

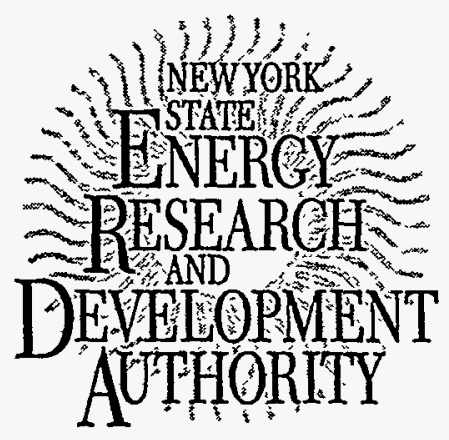




\section{DISCLAIMER}

This report was prepared as an account of work sponsored by an agency of the United States Government. Neither the United States Government nor any agency thereof, nor any of their employees, make any warranty, express or implied, or assimes any legal liability or responsibility for the accuracy, completeness, or usefulness of any information, apparatus, product, or process disclosed, or represents that its use would not infringe privately owned rights. Reference herein to any specific commercial product, process, or service by trade name, trademark, manufacturer, or otherwise does not necessarily constitute or imply its endorsement, recommendation, or favoring by the United States Government or any agency thereof. The views and opinions of authors expressed herein do not necessarily state or reflect those of the United States Government or any agency thereof. 


\section{DISCLAIMER}

Portions of this document may be illegible in electronic image products. Images are produced from the best available original document. 


\section{PATTERNS AND TRENDS \\ New York State Energy Profiles: 1980-1994}

\section{Table of Contents}

Introduction $\ldots \ldots \ldots \ldots \ldots \ldots \ldots \ldots \ldots \ldots \ldots \ldots \ldots \ldots \ldots \ldots \ldots \ldots$

Summary of New York State Energy Trends $\ldots \ldots \ldots \ldots \ldots \ldots \ldots \ldots$

Section $1 \quad$ Energy Profiles for the United States and New York State, $1994 \ldots \ldots \ldots 7$

Section 2 New York State Energy Consumption, 1980-1994 ........... 11

Section $3 \quad$ New York State Energy Prices, $1980-1994 \ldots \ldots \ldots \ldots \ldots \ldots . \ldots 23$

Section $4 \quad$ New York State Energy Expenditures, 1980-1994 . . . . . . . . . . 29

Section $5 \quad$ Sources of Energy Supplies, 1980-1994 . . . . . . . . . . . 33

Appendix A New York State Heating and Cooling Degree-Days, 1980-1994 . . . . . 37

Appendix B Abbreviations and Conversion Factors ............... 39

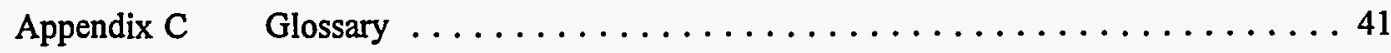

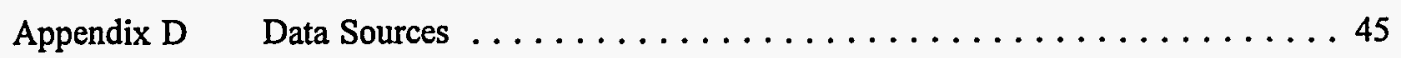

New York State Energy Profiles: 1980-1994 presents an overview of energy statistics for the State. It is intended to be an objective and reliable source of energy-related information for use by the general public, business, and government analysts. This report was prepared by using the most recent comprehensive data available covering the 1994 calendar year.

Questions should be directed to Tom Collins, NYSERDA's Manager of Technical Communications, at (518) 465-6251, ext. 250. 


\section{List of Tables and Figures}

Section 1 Energy Profiles for the United States and New York State, 1994

1-1 United States Primary Consumption by Fuel Type and Sector $\ldots \ldots \ldots \ldots \ldots \ldots \ldots \ldots \ldots$

1-2 New York State Primary Consumption by Fuel Type and Sector $\ldots \ldots \ldots \ldots \ldots \ldots \ldots$

Section 2 New York State Energy Consumption, 1980-1994

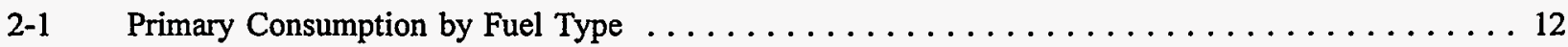

2-2 Primary Consumption of Refined Petroleum Products $\ldots \ldots \ldots \ldots \ldots \ldots \ldots \ldots \ldots \ldots$

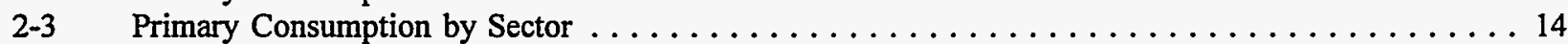

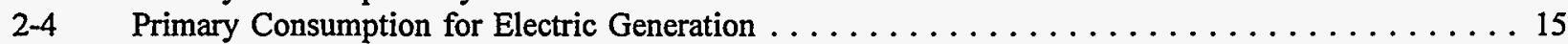

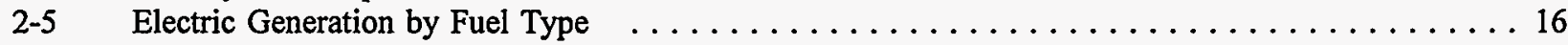

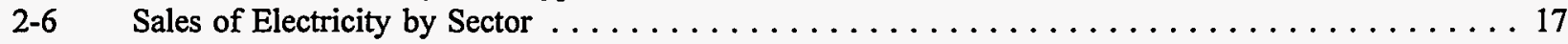

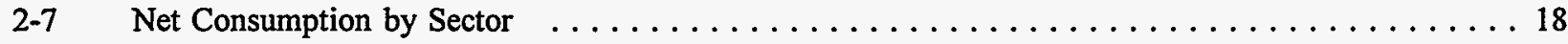

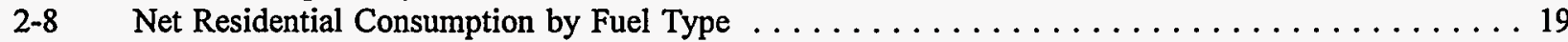

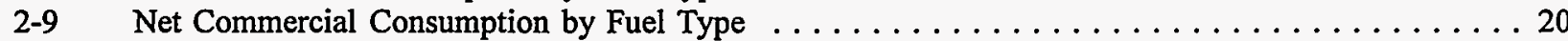

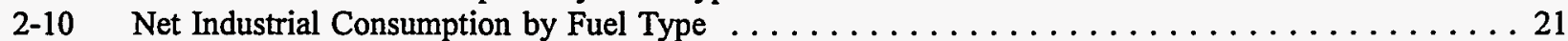

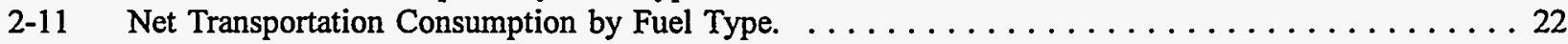

Section 3 New York State Retail Energy Prices (Nominal Dollars), 1980-1994

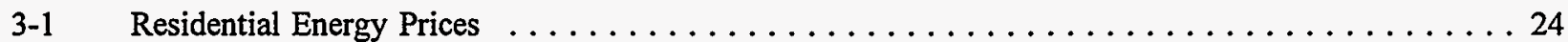

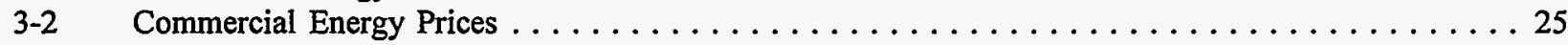

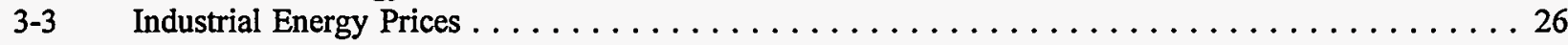

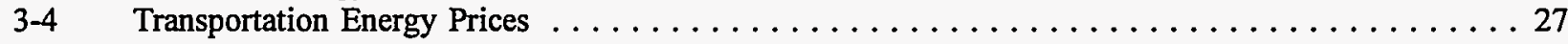

Section $4 \quad$ New York State Energy Expenditures, 1980-1994

4-1 Energy Expenditure Estimates by Fuel Type and Sector

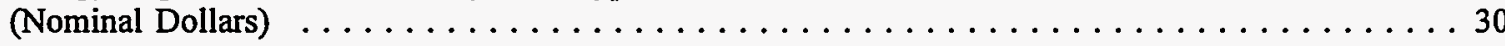

4-2 Energy Expenditure Estimates by Fuel Type and Sector

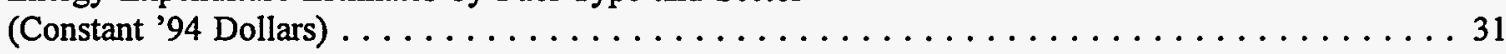

Section $5 \quad$ Sources of Energy Supplies, 1980-1994

5-1 Primary Energy Production for New York State, $1980-1994 \ldots \ldots \ldots \ldots \ldots \ldots \ldots \ldots \ldots$

5-2 Sources of Petroleum Products for

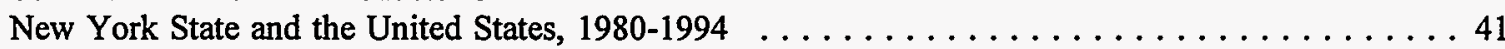

Appendix A New York State Degree-Days, 1980-1994

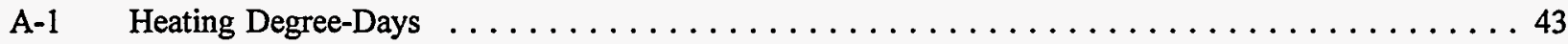

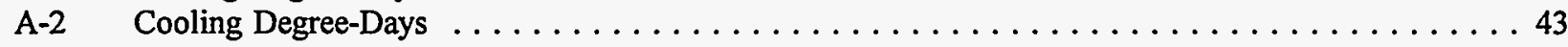




\section{INTRODUCTION}

The New York State Energy Research and Development Authority's Energy Analysis Program provides public and private sector stakeholders with useful independent and objective energy information.

This report provides an overview of one-year and 10-year, energy use trends in New York State. The information presented reflects energy consumption, supply, prices, and expenditures.

Section 1 is a 1994 overview of the United States and New York State energy profiles. The national energy consumption data used for comparison are compatible with New York State's data.

Section 2 provides current and historic data for primary and net consumption of energy by fuel type and sector. "Primary" represents total consumption of fuels by the residential, commercial, industrial, and transportation sectors including fuels used for generating electricity. "Net" is the end-use consumption by the residential, commercial, industrial, and transportation sectors, including electricity sales to each of these sectors but excluding energy losses incurred during electricity generation and distribution.

Section 3 presents energy price data at the retail level from 1980 to 1994 . Retail energy prices are provided by fuel type for the residential, commercial, industrial, and transportation sectors in nominal dollars cost per physical unit and per million Btu.

Section 4 presents the estimated cost of net energy consumption by sector and fuel type in nominal dollars and in 1994 constant dollars. Estimated costs were derived by multiplying quantities of consumption by their respective prices.

Section 5 details sources of New York State energy supplies. 


\section{SUMMARY OF NEW YORK STATE ENERGY TRENDS}

This section provides an overview of one-year and 10-year trends in energy consumption, supply, price, and expenditure by fuel type and sector for New York State.

\section{OVERVIEW}

1984-1994 Total primary energy consumption in 1994 was 3,880 trillion British thermal units (TBtu), 10\% above the 1984 consumption figure of $3,533 \mathrm{TBtu}$.

- Petroleum represented $41 \%$ of total primary energy consumption in 1994 , compared to $50 \%$ in 1984; natural gas usage increased from $23 \%$ to $30 \%$ over this 10 -year period.

- New York State's energy bill in nominal dollars totaled $\$ 32.4$ billion in 1994 , an increase of $18 \%$ over the $\$ 27.5$ billion spent in 1984 .

- While New York State is the fourth largest energy consumer among all states, only an estimated $7.9 \%$ of total energy requirements are met from resources provided within the State.

1993-1994 - Total primary consumption of energy in New York State decreased 1\% from 1993 to 1994.

- Consumption of coal, petroleum products, hydropower, and net imports of electricity all decreased; natural gas consumption and nuclear electricity generation increased.

- New York State's energy bill in nominal dollars of $\$ 32.4$ billion in 1994 was slightly higher than the $\$ 32.2$ billion spent in 1993.

- New York State ranks sixth among all states for the highest average price paid for energy.

\section{REVIEW BY FUEL TYPE}

\section{PEIROLEUM}

1984-1994 - Total annual demand for petroleum products in 1994 was 1,595 TBtu, 8\%, or 25 million barrels, below that of 1984, despite annual increases from 1986 to 1989.

- From 1984 to 1994, demand for residual fuel oil declined by 44\%; motor-gasoline demand declined $1 \%$. Conversely, demand for distillate, kerosene, and aviation fuel increased by $16 \%, 7 \%$, and $7 \%$, respectively.

- New York State's foreign oil dependence increased from $60 \%$ of the State's $\cdot$ total petroleum consumption in 1984 to $79 \%$ in 1994.

1993-1994 Consumption of total petroleum products decreased by 17 million barrels, or $6 \%$, from 1993 to 1994.

- Consumption of residual fuel oil, distillate oil, kerosene, and motor gasoline decreased by $17 \%, 7 \%, 6 \%$, and $3 \%$, respectively, between 1993 and 1994; aviation fuel consumption increased by $1 \%$. 
- Petroleum products accounted for $41 \%$ of the State's total energy requirements in 1994 , down from $43 \%$ in 1993.

- The State's reliance on foreign oil as a proportion of total petroleum consumption increased from $78 \%$ in 1993 to $79 \%$ in 1994 . OPEC's share of total oil consumption decreased slightly from $46 \%$ in 1993 to $45 \%$ in 1994 .

\section{NATURAL GAS}

1984-1994

- Sales of natural gas during 1994 (1,142 billion cubic feet) were $47 \%$ greater than in 1984 (774 billion cubic feet).

- Natural gas sales to commercial customers increased by $41 \%$ in 1994 from 163 billion cubic feet in 1984.

1993-1994 - Natural gas accounted for 30\% of New York State's total primary energy consumption in 1994 , compared to $27 \%$ in 1993.

- Between 1993 and 1994, sales of natural gas increased 9\%.

\section{COAL}

1984-1994 Coal use increased by 5\% between 1984 and 1994.

1993-1994 - Coal consumption in 1994 was 3\% below the 1993 level.

\section{HYDRO}

1984-1994 - Hydroelectricity generation in 1994 was about the same as it was in 1984.

1993-1994 Hydroelectricity generation in 1994 was 6\% below the 1993 level.

\section{NUCLEAR}

1984-1994 - Electricity generated by nuclear power plants was 38\% higher in 1994 than in 1984 .

1993-1994 - Nuclear electricity generation increased by 9\% from 1993 to 1994.

\section{NET IMPORTED ELECTRICITY}

1984-1994 - Net imported electricity in 1994 was $6 \%$ above the 1984 level.

1993-1994 - Net imported electricity decreased by 19\% from 1993 to 1994. 


\section{SECTOR REVIEW}

\section{RESIDENTIAL SECTOR}

1984-1994 - Total residential energy demand was 709 TBtu in 1994, 8\% above the 1984 figure.

- Demand for home heating oil declined $12 \%$ over the $1984-1994$ period.

- 1994 natural gas sales were 44 billion cubic feet, $13 \%$ higher than in 1984.

- Sales of electricity in 1994 were $23 \%$ greater than in 1984.

- The share of total residential energy use represented by petroleum products decreased from $30 \%$ in 1984 to $25 \%$ in 1994; natural gas consumption increased from $52 \%$ to $55 \%$ and electricity use increased from $17 \%$ to $19 \%$.

- New Yorkers spent in nominal dollars $\$ 10.4$ billion for the energy used in their homes during 1994, or $32 \%$ of the total end-use energy bill. The 1994 figure was $32 \%$ higher than the $\$ 7.9$ billion spent in 1984 .

- Between 1984 and 1994, average home-heating oil prices declined from $\$ 1.15$ to $\$ 1.00$ /gallon; natural gas prices increased $14 \%$, from $\$ 7.67$ to $\$ 8.73 /$ thousand cubic feet; and electricity prices increased $27 \%$, from 11.05 cents to 13.98 cents/kilowatt-hour.

1993-1994 In 1994, the residential sector represented $26 \%$ of total end-use energy consumption.

- Total residential energy consumption declined by $2 \%$ between 1993 and 1994. During 1994, Statewide heating degree-days were 1\% below the 1993 level; Statewide cooling degree-days were $2 \%$ lower than the 1993 level.

- The demand for distillate fuel oil declined 7\% from 1993 to 1994 , although sales of electricity and natural gas to residential customers each rose by $1 \%$.

- New Yorkers' expenditures in nominal dollars for energy used in their homes rose $3 \%$, from $\$ 10.1$ billion in 1993 to $\$ 10.4$ billion in 1994 .

- Between 1993 and 1994 the nominal price of home heating oil decreased by $2 \%$, natural gas rose by $7 \%$, and electricity increased by $3 \%$.

\section{COMMERGIAL SECTOR}

1984-1994 - Total consumption in the commercial sector was 651 TBtu in 1994, or $31 \%$ greater than the 1984 level of 499 TBtu.

- Sales of natural gas in 1994 were $41 \%$ above the 1984 level; consumption of petroleum products and electricity sales were $27 \%$ and $26 \%$ above 1984 levels, respectively.

- Natural gas, which accounted for $34 \%$ of total commercial energy demand in 1984 , increased to $37 \%$ in 1994 . The share represented by petroleum products decreased from $31 \%$ to $30 \%$; electricity's share decreased from $34 \%$ to $33 \%$.

- The energy bill in nominal dollars paid by commercial customers rose from $\$ 7.3$ billion in 1984 to $\$ 9.6$ billion in 1994 , an increase of $31 \%$. 
- The commercial sector's share of the total end-use energy bill was $27 \%$ in 1984 and $30 \%$ in 1994.

- From 1984 to 1994 , the nominal prices of distillate and residual fuel oil decreased by $25 \%$ and $40 \%$, respectively, while the nominal cost of electricity increased $8 \%$.

1993-1994 - In 1994, the commercial sector represented $24 \%$ of total end-use energy consumption.

- Commercial customers' demand for energy increased by 3\% between 1993 and 1994.

- The commercial sector's demand for petroleum products decreased by $7 \%$, sales of electricity increased by $1 \%$, and natural gas sales rose $16 \%$.

- The total energy bill in nominal dollars paid by commercial customers in 1994 was $3 \%$ higher than in 1993.

- Between 1993 and 1994, the nominal cost of natural gas for commercial customers rose by $1 \%$.

\section{INDUSTRIAL SECTOR}

1984-1994 - Industrial energy demand was $335 \mathrm{TBtu}$ in 1994, 13\% below the 1984 level.

- Coal consumption in the industrial sector fell by $40 \%$ from 1984 to 1994 , with coal's share of total energy consumption declining from $27 \%$ to $19 \%$ for the same period.

- Petroleum products, which constituted $19 \%$ of total industrial demand in 1984 , accounted for $13 \%$ in 1994; however, natural gas increased from $29 \%$ to $42 \%$, and electricity from $24 \%$ to $26 \%$.

- In nominal dollars industrial customers paid $\$ 2.8$ billion for energy in 1994 , roughly the same as in 1984.

- In 1994, industrial energy expenditures in nominal dollars were $9 \%$ of New York's total end-use energy bill, compared to $10 \%$ in 1984 .

- The nominal price of distillate fuel oil decreased by $29 \%$, while the residual fuel oil price decreased by $40 \%$.

- The nominal price of electricity increased by $17 \%$ over the 10 -year period.

1993-1994 - In 1994, industrial customers accounted for $12 \%$ of total end-use energy consumption.

- Industrial customers used 3\% less energy in 1994 than in 1993.

- From 1993 to 1994 , consumption of petroleum products decreased $21 \%$, sales of natural gas increased $2 \%$, electricity sales fell by $1 \%$, and coal consumption declined by $2 \%$.

- The energy bill in nominal dollars paid by industrial customers in 1994 decreased by $3 \%$ from 1993.

- Between 1993 and 1994, the nominal prices of natural gas and distillate fuel oil declined by $7 \%$ and $5 \%$, respectively. The nominal price of residual fuel oil showed a $5 \%$ increase; electricity increased by $1 \%$. 


\section{TRANSPORTATON SECTOR}

1984-1994 - Transportation sector energy demand was 1,072 TBtu in 1994, or 4\% above 1984 .

- Consumption of motor gasoline in 1994 was 4\% below that of 1984.

- The annual energy bill in nominal dollars for transportation reached $\$ 9.6$ billion in 1994, $30 \%$ of total end-use energy expenditures.

- From 1984 to 1994, average nominal retail gasoline prices (for all grades of gasoline) increased $2 \%$ from 124.9 cents to 127.6 cents per gallon; diesel fuels increased $2 \%$ from 133.6 cents to 136.8 cents per gallon. The price of residual fuels for bunkering decreased by $46 \%$ and jet fuel fell $33 \%$.

1993-1994 - In 1994, transportation accounted for 39\% of total end-use energy consumption.

- Between 1993 and 1994, energy consumption in the transportation sector declined $2 \%$.

- Consumption of diesel fuel and motor gasoline declined by $6 \%$ and $2 \%$, respectively, while aviation fuel consumption increased by $1 \%$.

- Energy expenditures in nominal dollars for the transportation sector decreased from $\$ 9.9$ billion in 1993 to $\$ 9.6$ billion in 1994 , or $3 \%$.

- Between 1993 and 1994, the nominal prices of motor gasoline and jet fuel decreased by $1 \%$ and $7 \%$, respectively. Vessel bunkering residual fuel and diesel fuel prices rose by $5 \%$ and $2 \%$, respectively.

\section{EleCtricity Generation}

1984-1994 - Consumption of energy for electricity generation represented $38 \%$ of primary consumption in 1984 and $40 \%$ in 1994.

- Consumption of energy for electricity generation rose by $16 \%$ between 1984 and 1994 .

- Petroleum product consumption decreased by 31 million barrels, or $62 \%$; natural gas use increased by 229 billion cubic feet, or $135 \%$ over the 1984 to 1994 period.

- Petroleum products share of fuel mix used to generate electricity decreased from $23 \%$ in 1984 to $7 \%$ in 1994.

- Natural gas share of fuel mix used to generate electricity increased from $13 \%$ in 1984 to $26 \%$ in 1994.

- Nuclear's share of fuel mix to generate electricity increased from $17 \%$ in 1984 to $20 \%$ in 1994; hydro's share declined from $21 \%$ to $18 \%$ for the same period.

1993-1994 - From 1993 to 1994, consumption of energy for electricity generation remained stable.

- Consumption of petroleum for electricity generation decreased by 5 million barrels, or $22 \%$, while natural gas use rose by 63 billion cubic feet, or $19 \%$.

- Nuclear generation increased by $9 \%$ between 1993 and 1994.

- Hydropower decreased by $6 \%$ from 1993 to 1994. 


\section{Section 1 \\ ENERGY PROFILES FOR THE UNITED STATES AND NEW YORK STATE}

This section presents an overview of the energy profiles for the United States and New York State in 1994. 
United States

Consumption of Energy by Fuel Type and Sector, 1994
Figure 1-1

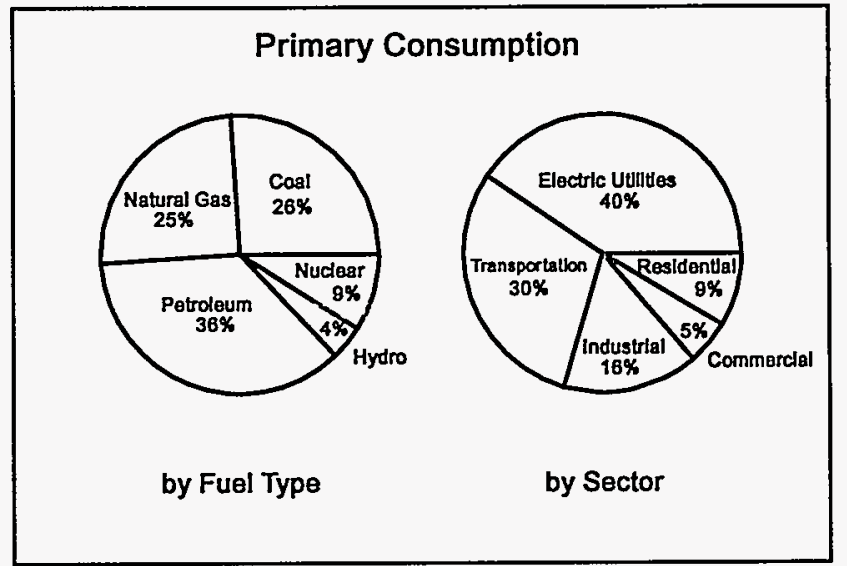

Table 1-1 (Trillion Btu)

\begin{tabular}{|c|c|c|c|c|c|c|c|}
\hline & $\frac{\text { Residential }}{\text { TBtu }}$ & $\begin{array}{r}\text { Commercial } \\
\text { TBtu }\end{array}$ & $\frac{\text { Industrial }}{\text { TBtu }}$ & $\frac{\text { Transportation }}{\text { TBtu }}$ & $\begin{array}{r}\text { Net } \\
\text { Consumption } \\
\text { TBtu }\end{array}$ & $\begin{array}{l}\text { Electric } \\
\text { Utilities' } \\
\text { TBtu }\end{array}$ & $\begin{array}{r}\begin{array}{r}\text { Primary } \\
\text { Consumption }\end{array} \\
\text { TBtu }\end{array}$ \\
\hline Coal & 47 & 95 & 2,510 & 0 & 2,652 & 16,910 & 19,562 \\
\hline Natural Gas & 4,980 & 3,093 & 7,590 & 0 & 15,663 & 3,056 & 18,719 \\
\hline Petroleum & 1,480 & 748 & 1,900 & 22,676 & 26,804 & 951 & 27,756 \\
\hline Distillate & 941 & 485 & 1,130 & 4,081 & 6,636 & 87 & 6,723 \\
\hline Residual & 0 & 163 & 420 & 887 & 1,471 & 864 & 2,335 \\
\hline Kerosene & 75 & 18 & 8 & 0 & 101 & 0 & 101 \\
\hline LPG & 464 & 82 & 342 & 28 & 916 & 0 & 916 \\
\hline Gasoline & 0 & 0 & 0 & 14,574 & 14,574 & 0 & 14,574 \\
\hline Jet Fuel & 0 & 0 & 0 & 3,105 & 3,105 & 0 & 3,105 \\
\hline Electric Sales & 3,432 & 3,135 & 3,386 & 13 & 9,966 & 20,733 & N/A \\
\hline $\begin{array}{r}\text { Net } \\
\text { Consumption }\end{array}$ & 9,938 & 7,071 & 15,386 & 22,689 & 55,085 & N/A & N/A \\
\hline & & & & \multicolumn{2}{|c|}{$\begin{array}{l}\text { Hydro Electricity }{ }^{2} \\
\text { Nuclear Electricity } \\
\text { Primary Consumption }\end{array}$} & $\begin{array}{r}2,941 \\
6,841 \\
30,699\end{array}$ & $\begin{array}{r}2,941 \\
6,841 \\
75,819\end{array}$ \\
\hline
\end{tabular}

1 Includes utility generators only.

2 Includes net imports of electricity. 
New York State

Consumption of Energy by Fuel Type and Sector, 1994
Figure 1-2

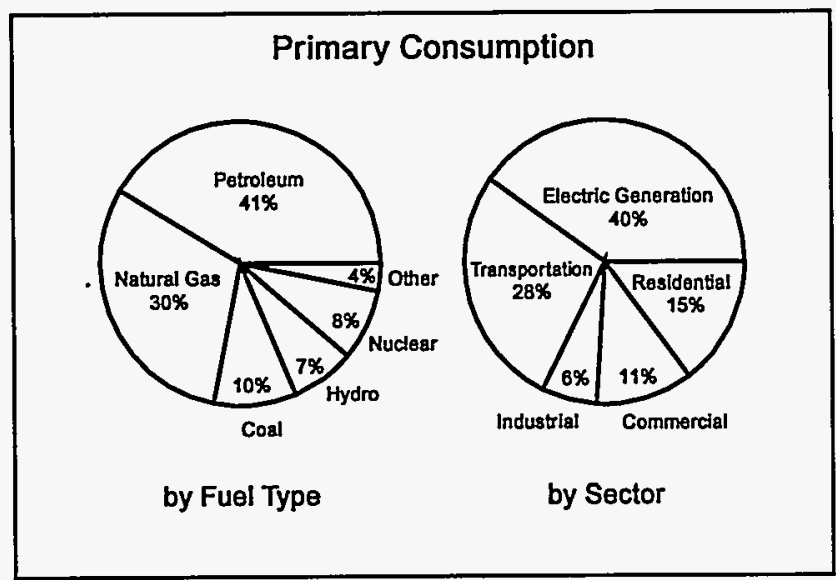

Table 1-2 (Trillion Btu)

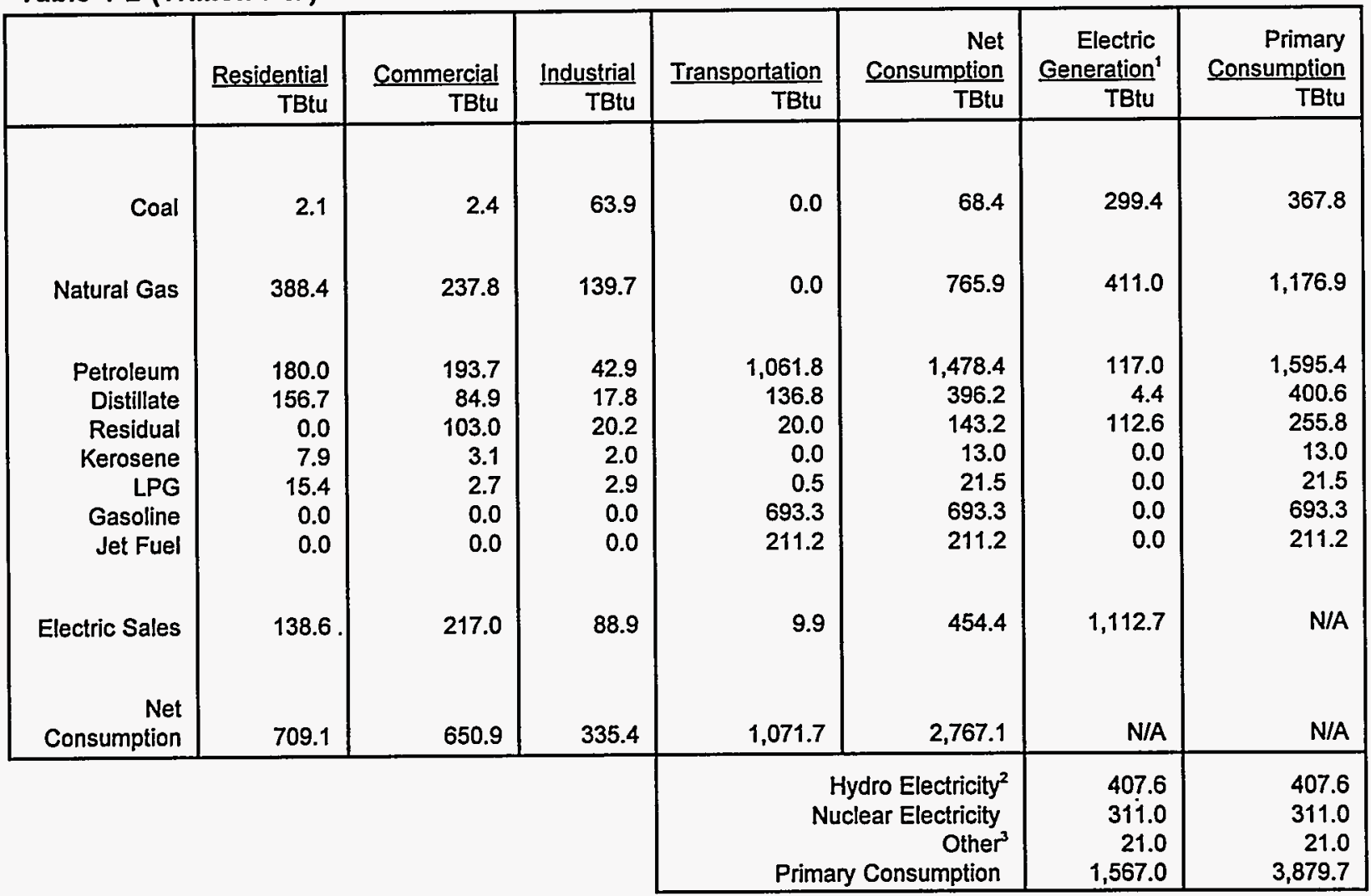

1 Includes utility and non-utility generators.

2 Includes net imports of electricity.

3 Includes renewable and indigenous fuels used by non-utility generators. 


\section{Section 2}

\section{NEW YORK STATE ENERGY CONSUMPTION}

This section presents data on primary and net consumption of energy in New York State by sector and fuel type for the period 1980 to 1994 . The 1994 data are preliminary estimates.

Primary consumption of energy is shown by fuel type in physical units and in British thermal units (Btu). Total primary consumption of energy by sector (residential, commercial, industrial, transportation, and electric generation) is presented for the 15-year period.

Electricity generation data are net of station use. Utility losses are primary inputs less the sales of electricity at 3,412 Btu per $\mathrm{kWh}$, and include generation and distribution losses, and statistical differences.

Hydro and nuclear electricity, as well as net electricity trade, have been converted to heat by application of an average annual heat rate (Btu per $\mathrm{kWh}$ generated) for coal-, oil-, and gas-fired power plants. This rate represents the replacement cost, in heat, of the electricity produced by these thermal plants.

All data relating to coal-fired generation include New York State Electric \& Gas Corporation's half-share of the Homer City Plant in Pennsylvania.

All end-use energy consumption by apartment buildings is included in the commercial sector.

Electricity sales are combined with the end-use consumption of coal, petroleum products, and natural gas to derive total net energy consumption in the residential, commercial, industrial, and transportation sectors. Total net energy consumption is provided in trillion Btu (TBtu) and in physical units such as tons, cubic feet, and barrels. 
New York State

Primary Consumption

by Fuel Type,

1980-1994
Figure 2-1

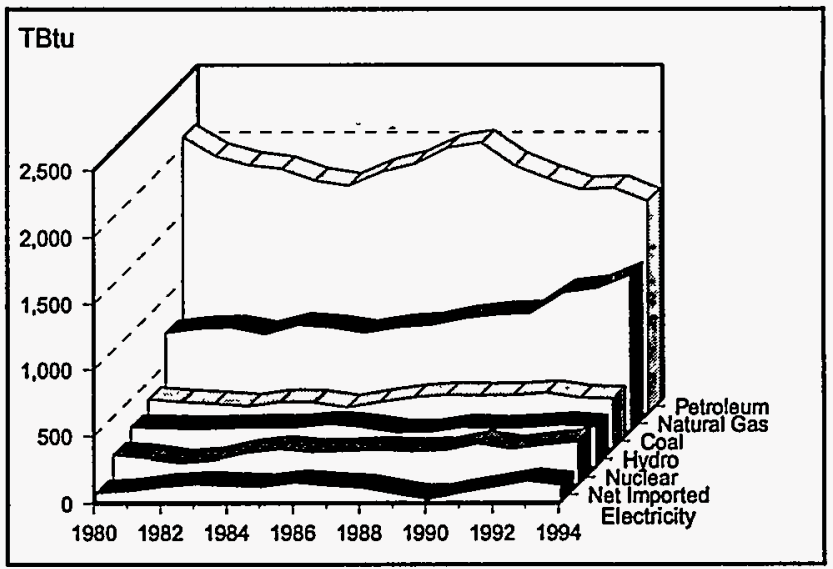

Table 2-1a (Physical Units)

\begin{tabular}{|c|c|c|c|c|c|c|c|}
\hline Year & $\begin{array}{r}\text { Coal } \\
\text { MTons }\end{array}$ & $\begin{array}{l}\text { Natural } \\
\text { Gas }^{i} \\
\text { MMcf }^{\prime}\end{array}$ & $\begin{array}{r}\text { Petroleum } \\
\text { Products } \\
\text { Mbbl }\end{array}$ & $\frac{\text { Hydro }}{\mathrm{GWh}}$ & $\frac{\text { Nuclear }}{\mathrm{GWh}}$ & $\begin{array}{r}\text { Net } \\
\text { Imported } \\
\text { Electricity } \\
\mathrm{GWh}\end{array}$ & $\frac{\text { Other }}{\mathrm{GWh}}$ \\
\hline 1980 & 14,952 & 719,146 & 364,105 & 26,241 & 19,276 & 5,575 & 0 \\
\hline 1981 & 14,183 & 748,807 & 339,520 & 25,658 & 17,444 & 7,137 & 0 \\
\hline 1982 & 13,521 & 758,716 & 328,958 & 25,329 & 14,438 & 10,196 & 0 \\
\hline 1983 & 12,782 & 707,988 & 324,770 & 26,162 & 16,376 & 11,888 & 0 \\
\hline 1984 & 14,109 & 774,486 & 311,165 & 26,586 & 21,187 & 10,812 & 0 \\
\hline 1985 & 13,948 & 753,777 & 305,950 & 26,956 & 24,092 & 10,155 & 0 \\
\hline 1986 & 12,202 & 715,909 & 323,270 & 29,480 & 22,084 & 13,204 & 0 \\
\hline 1987 & 13,795 & 757,211 & 334,252 & 27,546 & 22,926 & 11,373 & 0 \\
\hline 1988 & 15,076 & 774,455 & 353,850 & 23,994 & 24,176 & 9,994 & 0 \\
\hline 1989 & 15,857 & 821,436 & 360,310 & 23,918 & 22,846 & 5,539 & 0 \\
\hline 1990 & 15,572 & 851,590 & 331,193 & 27,560 & 23,624 & 1,352 & 1,502 \\
\hline 1991 & 15,658 & 860,739 & 314,723 & 26,257 & 28,477 & 6,323 & 1,622 \\
\hline 1992 & 16,272 & $1,015,693$ & 301,322 & 27,193 & 24,155 & 10,167 & 1,806 \\
\hline 1993 & 14,825 & $1,043,198$ & 303,666 & 28,479 & 26,889 & 14,280 & 1,980 \\
\hline 1994 & 14,378 & $1,141,545$ & 286,572 & 26,800 & 29,225 & 11,504 & 1,972 \\
\hline
\end{tabular}

Table 2-1b (Trillion Btu)

\begin{tabular}{|c|c|c|c|c|c|c|c|c|}
\hline Year & $\frac{\text { Coal }}{\text { TBtu }}$ & $\begin{array}{c}\text { Natural } \\
\frac{\text { Gas' }}{\text { TBtu }}\end{array}$ & $\begin{array}{r}\text { Petroleum } \\
\text { Products } \\
\text { TBtu }\end{array}$ & $\frac{\text { Hydro }}{\text { TBtu }}$ & $\frac{\text { Nuclear }}{\text { TBtu }}$ & $\begin{array}{r}\text { Net } \\
\text { Imported } \\
\text { Electricity } \\
\text { TBtu }\end{array}$ & $\frac{\text { Other }^{2}}{\text { TBtu }}$ & $\frac{\text { Totat }}{\text { TBtu }}$ \\
\hline 1980 & 369.2 & 737.9 & $2,081.2$ & 288.2 & 211.7 & 61.2 & 0.0 & $3,749.4$ \\
\hline 1981 & 347.4 & 768.3 & $1,935.7$ & 280.9 & 191.0 & 78.1 & 0.0 & $3,601,4$ \\
\hline 1982 & 333.4 & 779.1 & $1,869.2$ & 274.0 & 156.2 & 110.3 & 0.0 & $3,522.1$ \\
\hline 1983 & 314.9 & 729.9 & $1,838.6$ & 280.4 & 175.5 & 127.4 & 0.0 & $3,466.7$ \\
\hline 1984 & 349.0 & 798.1 & $1,751.8$ & 287.6 & 229.2 & 117.0 & 0.0 & $3,532.6$ \\
\hline 1985 & 344.6 & 778.2 & $1,714.0$ & 287.3 & 256.7 & 108.2 & 0.0 & $3,489.1$ \\
\hline 1986 & 305.5 & 737.6 & $1,819.1$ & 311.3 & 233.2 & 139.4 & 0.0 & $3,546.0$ \\
\hline 1987 & 348.5 & 780.2 & $1,878.7$ & 288.2 & 239.9 & 119.0 & 0.0 & $3,654.5$ \\
\hline 1988 & 382.5 & 796.9 & $1,998.7$ & 247.6 & 249.4 & 103.1 & 0.0 & $3,778.2$ \\
\hline 1989 & 401.5 & 846.4 & $2,037.1$ & 250.0 & 238.8 & 57.9 & 0.0 & $3,831.5$ \\
\hline 1990 & 394.8 & 877.8 & $1,865.9$ & 288.0 & 246.9 & 14.1 & 15.7 & $3,703.3$ \\
\hline 1991 & 398.6 & 887.7 & $1,766.8$ & 274.2 & 297.4 & 66.0 & 16.9 & $3,707.7$ \\
\hline 1992 & 416.4 & $1,046.2$ & $1,682.2$ & 287.9 & 255.7 & 107.6 & 19.1 & $3,815.2$ \\
\hline 1993 & 377.6 & $1,075.5$ & $1,695.9$ & 303.6 & 286.6 & 152.2 & 21.1 & $3,912,6$ \\
\hline 1994 & 367.8 & $1,176.9$ & $1,595.4$ & 285.2 & 311.0 & 122.4 & 21.0 & $3,879.7$ \\
\hline
\end{tabular}

${ }^{1}$ Excludes lease, plant and pipeline fuels.

${ }^{2}$ Includes renewable and indigenous energy.

${ }^{3}$ Excludes non-fuel uses and steam. 
New York State

Primary Consumption

of Refined Petroleum Products, 1980-1994
Figure 2-2

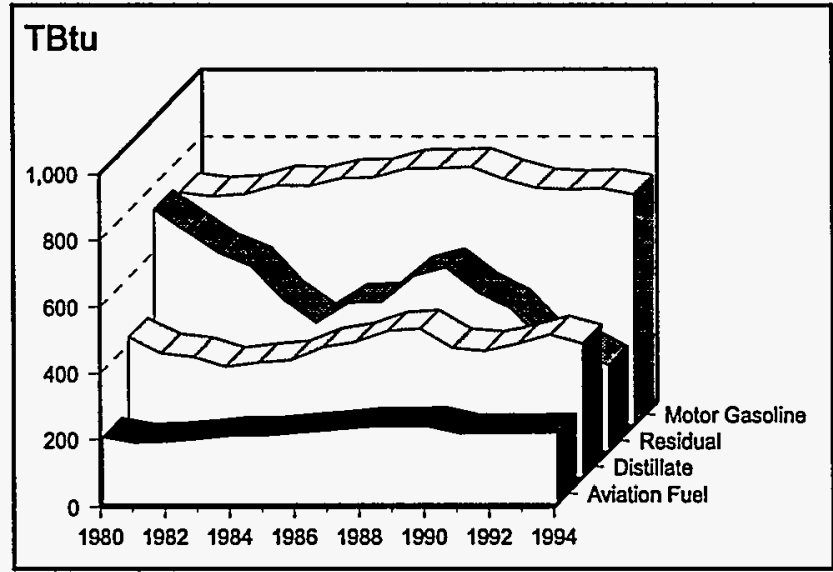

Table 2-2a (Thousand Barrels)

\begin{tabular}{|c|c|c|c|c|c|c|c|}
\hline Year & $\frac{\text { Distillate }}{\mathrm{Mbbl}}$ & $\frac{\text { Residual }}{\mathrm{Mbbl}}$ & $\frac{\text { Kerosene }}{\mathrm{Mbbl}}$ & $\frac{\mathrm{LPG}^{1}}{\mathrm{Mbbl}}$ & $\begin{array}{r}\text { Motor } \\
\text { Gasoline } \\
\text { Mbbl }\end{array}$ & $\begin{array}{l}\text { Aviation } \\
\text { Euels }^{2} \\
\text { Mbbl }\end{array}$ & $\begin{array}{r}\text { Total } \\
\text { Petroleum } \\
\text { Products } \\
\text { Mbbl }\end{array}$ \\
\hline $\begin{array}{l}1980 \\
1981 \\
1982 \\
1983 \\
1984\end{array}$ & $\begin{array}{l}72,340 \\
64,112 \\
62,154 \\
57,136 \\
59,217\end{array}$ & $\begin{array}{r}115,735 \\
105,212 \\
94,732 \\
88,438 \\
72,312\end{array}$ & $\begin{array}{l}2,309 \\
2,072 \\
2,561 \\
3,007 \\
2,149\end{array}$ & $\begin{array}{l}5,631 \\
5,215 \\
4,877 \\
4,906 \\
5,055\end{array}$ & $\begin{array}{l}133,282 \\
131,037 \\
132,218 \\
137,445 \\
136,788\end{array}$ & $\begin{array}{l}34,808 \\
31,872 \\
32,416 \\
33,838 \\
35,644\end{array}$ & $\begin{array}{l}364,105 \\
339,520 \\
328,958 \\
324,770 \\
311,165\end{array}$ \\
\hline $\begin{array}{l}1985 \\
1986 \\
1987 \\
1988 \\
1989\end{array}$ & $\begin{array}{l}60,399 \\
67,090 \\
69,846 \\
75,350 \\
76,545\end{array}$ & $\begin{array}{l}61,395 \\
70,961 \\
71,183 \\
83,656 \\
87,682\end{array}$ & $\begin{array}{l}2,411 \\
1,552 \\
2,382 \\
2,431 \\
2,344\end{array}$ & $\begin{array}{l}4,923 \\
4,878 \\
5,476 \\
5,238 \\
5,579\end{array}$ & $\begin{array}{l}141,249 \\
141,699 \\
146,758 \\
147,000 \\
147,800\end{array}$ & $\begin{array}{l}35,573 \\
37,090 \\
38,607 \\
40,175 \\
40,360\end{array}$ & $\begin{array}{l}305,950 \\
323,270 \\
334,252 \\
353,850 \\
360,310\end{array}$ \\
\hline $\begin{array}{l}1990 \\
1991 \\
1992 \\
1993 \\
1994\end{array}$ & $\begin{array}{l}66,719 \\
65,277 \\
68,903 \\
73,511 \\
68,766\end{array}$ & $\begin{array}{l}75,784 \\
68,076 \\
52,011 \\
49,295 \\
40,697\end{array}$ & $\begin{array}{l}1,504 \\
1,753 \\
1,861 \\
2,421 \\
2,289\end{array}$ & $\begin{array}{l}5,606 \\
7,207 \\
7,077 \\
6,139 \\
5,582\end{array}$ & $\begin{array}{l}140,900 \\
135,660 \\
134,600 \\
135,350 \\
131,988\end{array}$ & $\begin{array}{l}40,680 \\
36,750 \\
36,870 \\
36,950 \\
37,250\end{array}$ & $\begin{array}{l}331,193 \\
314,723 \\
301,322 \\
303,666 \\
286,572\end{array}$ \\
\hline
\end{tabular}

Table 2-2b (Trillion Btu)

\begin{tabular}{|c|c|c|c|c|c|c|c|}
\hline Year & $\frac{\text { Distillate }}{\text { TBtu }}$ & $\frac{\text { Residual }}{\text { TBtu }}$ & $\frac{\text { Kerosene }}{\text { TBtu }}$ & $\frac{\text { LPG }^{1}}{\text { TBtu }}$ & $\begin{array}{r}\text { Motor } \\
\text { Gasoline } \\
\text { TBtu }\end{array}$ & $\begin{array}{l}\text { Aviation } \\
\frac{\text { Fuels }}{2} \\
\text { TBtu }\end{array}$ & $\begin{array}{r}\text { Total } \\
\text { Petroleum } \\
\text { Products } \\
\text { TBtu }\end{array}$ \\
\hline $\begin{array}{l}1980 \\
1981 \\
1982 \\
1983 \\
1984\end{array}$ & $\begin{array}{l}421.4 \\
373.5 \\
362.0 \\
332.8 \\
344.9\end{array}$ & $\begin{array}{l}727.6 \\
661.5 \\
595.6 \\
556.0 \\
454.6\end{array}$ & $\begin{array}{l}13.1 \\
11.7 \\
14.5 \\
17.0 \\
12.2\end{array}$ & $\begin{array}{l}21.6 \\
20.0 \\
18.7 \\
18.8 \\
19.4\end{array}$ & $\begin{array}{l}700.1 \\
688.3 \\
694.5 \\
722.0 \\
718.5\end{array}$ & $\begin{array}{l}197.4 \\
180.7 \\
183.8 \\
191.9 \\
202.1\end{array}$ & $\begin{array}{l}2,081.2 \\
1,935.7 \\
1,869.2 \\
1,838.6 \\
1,751.8\end{array}$ \\
\hline $\begin{array}{l}1985 \\
1986 \\
1987 \\
1988 \\
1989\end{array}$ & $\begin{array}{l}351.8 \\
390.8 \\
406.9 \\
438.9 \\
445.9\end{array}$ & $\begin{array}{l}386.0 \\
446.1 \\
447.5 \\
525.9 \\
551.3\end{array}$ & $\begin{array}{r}13.7 \\
8.8 \\
13.5 \\
13.8 \\
13.3\end{array}$ & $\begin{array}{l}18.9 \\
18.7 \\
21.0 \\
20.1 \\
21.4\end{array}$ & $\begin{array}{l}742.0 \\
744.3 \\
770.9 \\
772.2 \\
776.4\end{array}$ & $\begin{array}{l}201.7 \\
210.3 \\
218.9 \\
227.8 \\
228.8\end{array}$ & $\begin{array}{l}1,714.0 \\
1,819.1 \\
1,878.7 \\
1,998.7 \\
2,037.1\end{array}$ \\
\hline $\begin{array}{l}1990 \\
1991 \\
1992 \\
1993 \\
1994\end{array}$ & $\begin{array}{l}388.6 \\
380.2 \\
401.4 \\
428.2 \\
400.6\end{array}$ & $\begin{array}{l}476.5 \\
428.0 \\
327.0 \\
309.9 \\
255.9\end{array}$ & $\begin{array}{r}8.5 \\
9.9 \\
10.6 \\
13.7 \\
13.0\end{array}$ & $\begin{array}{l}21.5 \\
27.6 \\
27.1 \\
23.5 \\
21.4\end{array}$ & $\begin{array}{l}740.1 \\
712.6 \\
707.1 \\
711.0 \\
693.3\end{array}$ & $\begin{array}{l}230.7 \\
208.4 \\
209.1 \\
209.5 \\
211.2\end{array}$ & $\begin{array}{l}1,865.9 \\
1,766.8 \\
1,682.2 \\
1,695.9 \\
1,595.4\end{array}$ \\
\hline
\end{tabular}

${ }^{1}$ Excludes non-fuel uses.

${ }^{2}$ Kerosene-type jet fuel and aviation gasoline. 
New York State

Primary Consumption

by Sector,

1980-1994
Figure 2-3

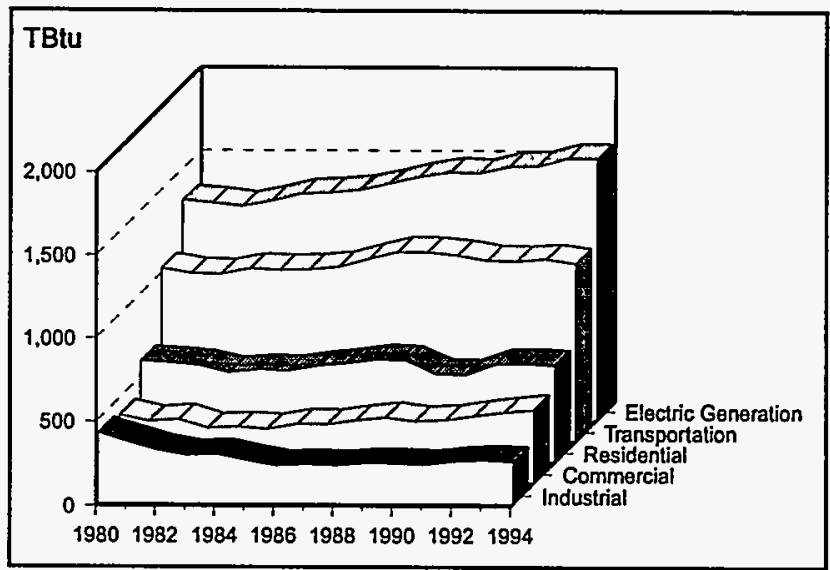

Table 2-3 (Trillion Btu)

\begin{tabular}{|r|r|r|r|r|r|r|}
\hline Year & Residential & Commercial & Tndustrial & Transportation & $\begin{array}{r}\text { Electric } \\
\text { Teneration } \\
\text { TBtu }\end{array}$ & $\frac{\text { TBtu }}{\text { Total }}$ \\
\hline 1980 & 594.6 & 398.3 & 418.1 & $1,027.8$ & $1,310.7$ & $3,749.4$ \\
1981 & 584.9 & 360.1 & 364.4 & 998.0 & $1,294.0$ & $3,601.4$ \\
1982 & 568.2 & 373.6 & 314.3 & 993.1 & $1,272.8$ & $3,522.1$ \\
1983 & 528.6 & 319.5 & 283.3 & $1,028.0$ & $1,307.2$ & $3,466.7$ \\
1984 & 544.5 & 326.9 & 291.7 & $1,019.8$ & $1,349.7$ & $3,532.6$ \\
\hline 1985 & 537.9 & 316.0 & 253.1 & $1,024.6$ & $1,357.5$ & $3,489.1$ \\
1986 & 564.9 & 346.5 & 222.4 & $1,040.0$ & $1,372.2$ & $3,546.0$ \\
1987 & 581.8 & 344.0 & 229.9 & $1,087.0$ & $1,411.7$ & $3,654.5$ \\
1988 & 605.5 & 368.9 & 222.6 & $1,129.2$ & $1,452.0$ & $3,778.2$ \\
1989 & 600.0 & 385.8 & 237.3 & $1,128.4$ & $1,480.2$ & $3,831.5$ \\
\hline 1990 & 521.7 & 363.8 & 234.9 & $1,109.0$ & $1,473.9$ & $3,703.3$ \\
1991 & 516.1 & 370.6 & 226.9 & $1,079.0$ & $1,515.1$ & $3,707.7$ \\
1992 & 577.9 & 395.9 & 247.0 & $1,074.7$ & $1,519.7$ & $3,815.2$ \\
1993 & 582.8 & 417.4 & 255.8 & $1,088.4$ & $1,568.2$ & $3,912.6$ \\
1994 & 570.5 & 433.9 & 246.4 & $1,061.8$ & $1,567.1$ & $3,879.7$ \\
\hline
\end{tabular}


New York State

Primary Consumption

for Electric Generation

1980-1994
Figure 2-4

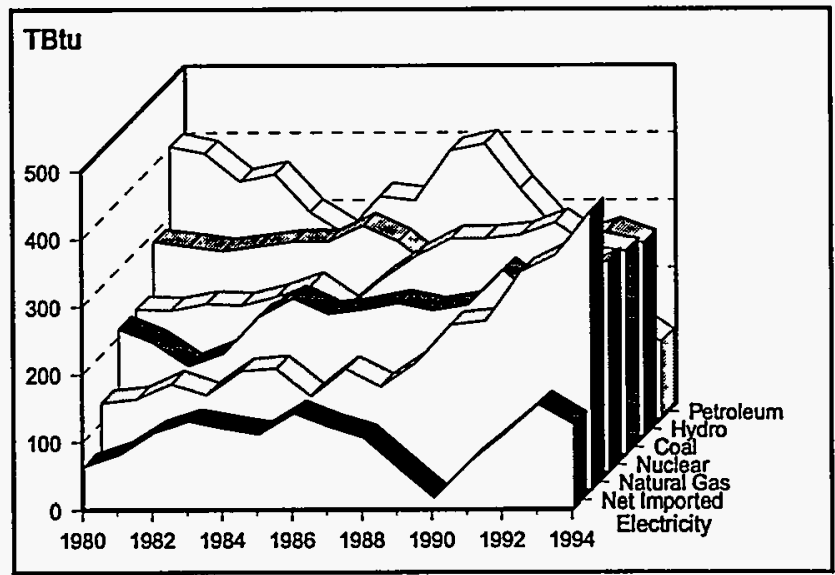

Table 2-4a (Physical Units)

\begin{tabular}{|c|c|c|c|c|c|c|c|c|c|}
\hline Year & $\begin{array}{r}\text { Coal' } \\
\text { MTons }\end{array}$ & $\begin{array}{r}\text { Natural } \\
\text { Gas } \\
\text { MMcf }\end{array}$ & $\frac{\text { Distillate }^{2}}{\mathrm{Mbbl}}$ & $\frac{\text { Residual }}{\mathrm{Mbbl}}$ & $\begin{array}{r}\begin{array}{r}\text { Total } \\
\text { Petroleum }\end{array} \\
\mathbf{M b b l}\end{array}$ & $\frac{\text { Hydro }}{\text { GWh }}$ & $\frac{\text { Nuclear }}{\text { GWh }}$ & $\begin{array}{r}\text { Net } \\
\text { Imported } \\
\text { Electricity } \\
\text { GWh }\end{array}$ & $\frac{\text { Other }}{\text { GWh }}$ \\
\hline $\begin{array}{l}1980 \\
1981 \\
1982 \\
1983 \\
1984 \\
\end{array}$ & $\begin{array}{l}8,709 \\
8,706 \\
8,946 \\
8,802 \\
9,247 \\
\end{array}$ & $\begin{array}{l}124,391 \\
130,507 \\
152,577 \\
135,811 \\
169,620\end{array}$ & $\begin{array}{l}585 \\
746 \\
500 \\
433 \\
617 \\
\end{array}$ & $\begin{array}{l}64,062 \\
62,419 \\
55,686 \\
57,531 \\
48,548 \\
\end{array}$ & $\begin{array}{l}64,647 \\
63,165 \\
56,186 \\
57,964 \\
49,165\end{array}$ & $\begin{array}{l}26,241 \\
25,658 \\
25,329 \\
26,162 \\
26,586 \\
\end{array}$ & $\begin{array}{l}19,276 \\
17,444 \\
14,438 \\
16,376 \\
21,187 \\
\end{array}$ & $\begin{array}{r}5,575 \\
7,137 \\
10,196 \\
11,888 \\
10,812 \\
\end{array}$ & $\begin{array}{l}0 \\
0 \\
0 \\
0 \\
0\end{array}$ \\
\hline $\begin{array}{l}1985 \\
1986 \\
1987 \\
1988 \\
1989\end{array}$ & $\begin{array}{r}9,905 \\
8,430 \\
10,039 \\
11,380 \\
12,432\end{array}$ & $\begin{array}{l}172,631 \\
133,532 \\
173,328 \\
148,186 \\
182,002\end{array}$ & $\begin{array}{r}623 \\
1,166 \\
1,262 \\
2,019 \\
3,525\end{array}$ & $\begin{array}{l}43,418 \\
52,288 \\
51,276 \\
62,983 \\
64,747\end{array}$ & $\begin{array}{l}44,041 \\
53,454 \\
52,538 \\
65,002 \\
68,272\end{array}$ & $\begin{array}{l}26,956 \\
29,480 \\
27,546 \\
23,994 \\
23,918\end{array}$ & $\begin{array}{l}24,092 \\
22,084 \\
22,926 \\
24,176 \\
22,846\end{array}$ & $\begin{array}{r}10,155 \\
13,204 \\
11,373 \\
9,994 \\
5,539\end{array}$ & $\begin{array}{l}0 \\
0 \\
0 \\
0 \\
0\end{array}$ \\
\hline $\begin{array}{l}1990 \\
1991 \\
1992 \\
1993 \\
1994\end{array}$ & $\begin{array}{l}12,400 \\
12,532 \\
13,184 \\
11,907 \\
11,552\end{array}$ & $\begin{array}{l}235,909 \\
241,478 \\
312,516 \\
336,052 \\
398,674\end{array}$ & $\begin{array}{r}1,369 \\
1,241 \\
651 \\
432 \\
747\end{array}$ & $\begin{array}{l}53,903 \\
44,578 \\
28,963 \\
23,566 \\
17,917\end{array}$ & $\begin{array}{l}55,272 \\
45,819 \\
29,614 \\
23,998 \\
18,664\end{array}$ & $\begin{array}{l}27,560 \\
26,257 \\
27,193 \\
28,479 \\
26,800\end{array}$ & $\begin{array}{l}23,624 \\
28,477 \\
24,155 \\
26,889 \\
29,225\end{array}$ & $\begin{array}{r}1,352 \\
6,323 \\
10,167 \\
14,280 \\
11,504\end{array}$ & $\begin{array}{l}1,502 \\
1,622 \\
1,806 \\
1,980 \\
1,972\end{array}$ \\
\hline
\end{tabular}

Table 2-4b (Trillion Btu)

\begin{tabular}{|c|c|c|c|c|c|c|c|c|c|c|}
\hline Year & $\frac{\text { Coal' }}{\text { TBtu }}$ & $\begin{array}{r}\text { Natural } \\
\text { Gas } \\
\text { TBtu }\end{array}$ & $\frac{\text { Distillate }^{2}}{\text { TBtu }}$ & $\frac{\text { Residual }}{\text { TBtu }}$ & $\begin{array}{r}\begin{array}{r}\text { Total } \\
\text { Petroleum }\end{array} \\
\text { TBtu }\end{array}$ & $\frac{\text { Hydro }}{\text { TBtu }}$ & $\frac{\text { Nuclear }}{\text { TBtu }}$ & $\begin{array}{r}\text { Net } \\
\text { Imported } \\
\text { Electricity } \\
\text { TBtu }\end{array}$ & $\frac{\text { Other }^{3}}{\text { TBtu }}$ & $\frac{\text { Total }^{4}}{\text { TBtu }}$ \\
\hline $\begin{array}{l}1980 \\
1981 \\
1982 \\
1983 \\
1984\end{array}$ & $\begin{array}{l}214.5 \\
212.7 \\
222.2 \\
219.8 \\
232.1\end{array}$ & $\begin{array}{l}128.9 \\
134.6 \\
157.2 \\
140.0 \\
175.0\end{array}$ & $\begin{array}{l}3.4 \\
4.3 \\
2.9 \\
2.5 \\
3.6\end{array}$ & $\begin{array}{l}402.8 \\
392.4 \\
350.1 \\
361.7 \\
305.2\end{array}$ & $\begin{array}{l}406.2 \\
396.8 \\
353.0 \\
364.2 \\
308.8\end{array}$ & $\begin{array}{l}288.2 \\
280.9 \\
274.0 \\
280.4 \\
287.6\end{array}$ & $\begin{array}{l}211.7 \\
191.0 \\
156.2 \\
175.5 \\
229.2\end{array}$ & $\begin{array}{r}61.2 \\
78.1 \\
110.3 \\
127.4 \\
117.0\end{array}$ & $\begin{array}{l}0.0 \\
0.0 \\
0.0 \\
0.0 \\
0.0\end{array}$ & $\begin{array}{l}1,310.7 \\
1,294.0 \\
1,272.8 \\
1,307.2 \\
1,349.7\end{array}$ \\
\hline $\begin{array}{l}1985 \\
1986 \\
1987 \\
1988 \\
1989\end{array}$ & $\begin{array}{l}249.6 \\
214.5 \\
256.7 \\
291.7 \\
318.9\end{array}$ & $\begin{array}{l}179.0 \\
138.3 \\
178.2 \\
152.5 \\
187.1\end{array}$ & $\begin{array}{r}3.6 \\
6.8 \\
7.4 \\
11.8 \\
20.5\end{array}$ & $\begin{array}{l}273.0 \\
328.7 \\
322.4 \\
396.0 \\
407.1\end{array}$ & $\begin{array}{l}276.6 \\
335.5 \\
329.7 \\
407.7 \\
427.6\end{array}$ & $\begin{array}{l}287.3 \\
311.3 \\
288.2 \\
247.6 \\
250.0\end{array}$ & $\begin{array}{l}256.7 \\
233.2 \\
239.9 \\
249.4 \\
238.8\end{array}$ & $\begin{array}{r}108.2 \\
139.4 \\
119.0 \\
103.1 \\
57.9\end{array}$ & $\begin{array}{l}0.0 \\
0.0 \\
0.0 \\
0.0 \\
0.0\end{array}$ & $\begin{array}{l}1,357.5 \\
1,372.2 \\
1,411.7 \\
1,452.0 \\
1,480.2\end{array}$ \\
\hline $\begin{array}{l}1990 \\
1991 \\
1992 \\
1993 \\
1994\end{array}$ & $\begin{array}{l}318.6 \\
323.8 \\
342.2 \\
307.4 \\
299.4\end{array}$ & $\begin{array}{l}243.7 \\
249.2 \\
321.3 \\
346.5 \\
411.0\end{array}$ & $\begin{array}{l}8.0 \\
7.2 \\
3.8 \\
2.5 \\
4.4\end{array}$ & $\begin{array}{l}338.9 \\
280.3 \\
182.1 \\
148.2 \\
112.6\end{array}$ & $\begin{array}{l}346.9 \\
287.5 \\
185.9 \\
150.7 \\
117.0\end{array}$ & $\begin{array}{l}288.0 \\
274.2 \\
287.9 \\
303.6 \\
285.2\end{array}$ & $\begin{array}{l}246.9 \\
297.4 \\
255.7 \\
286.6 \\
311.0\end{array}$ & $\begin{array}{r}14.1 \\
66.0 \\
107.6 \\
152.2 \\
122.4\end{array}$ & $\begin{array}{l}15.7 \\
16.9 \\
19.1 \\
21.1 \\
21.0\end{array}$ & $\begin{array}{l}1,473.9 \\
1,515.1 \\
1,519.7 \\
1,568.2 \\
1,567.1\end{array}$ \\
\hline
\end{tabular}

Bituminous coal only.

2 Includes small quantities of kerosene-type jet fuel.

3 Includes renewable and indigenous fuels used by non-utility generators.

4 Excludes utility consumption of fuels used in the production of steam which is distributed for space heating. 
New York State

Electric Generation

by Fuel Type,

1980-1994
Figure 2-5

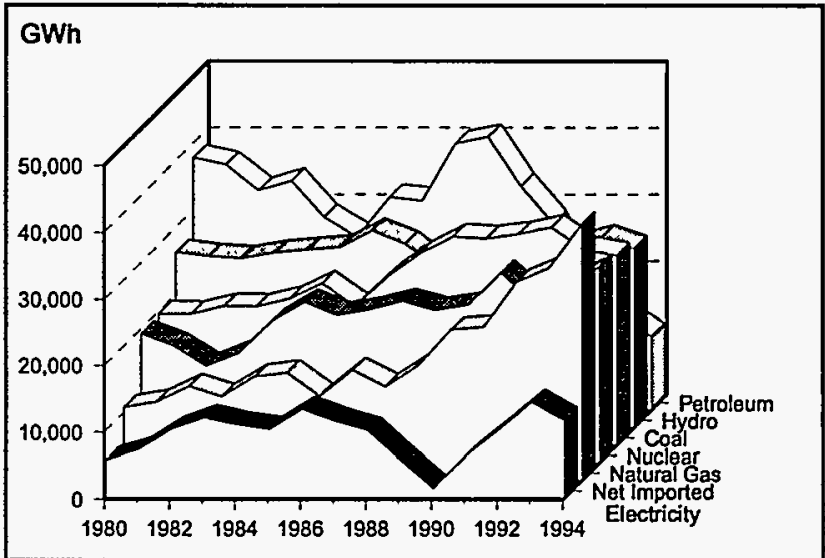

Table 2-5 (Gigawatthours)

\begin{tabular}{|c|c|c|c|c|c|c|c|c|}
\hline Year & $\frac{\text { Coal }}{\text { GWh }}$ & $\begin{array}{r}\text { Natural } \\
\frac{\text { Gas }}{\text { GWh }}\end{array}$ & $\begin{array}{r}\text { Petroleum } \\
\text { Products } \\
\text { GWh }\end{array}$ & $\frac{\text { Hydro }}{\text { GWh }}$ & $\frac{\text { Nuclear }}{\text { GWh }}$ & $\begin{array}{r}\text { Net } \\
\text { Imported } \\
\text { Electricity } \\
\text { GWh }\end{array}$ & $\frac{\text { Other }^{1}}{\text { GWh }}$ & $\frac{\text { Total }}{\text { GWh }}$ \\
\hline $\begin{array}{l}1980 \\
1981 \\
1982 \\
1983 \\
1984\end{array}$ & $\begin{array}{l}19,643 \\
19,632 \\
20,807 \\
20,753 \\
21,902\end{array}$ & $\begin{array}{l}10,766 \\
11,566 \\
13,974 \\
12,428 \\
15,395\end{array}$ & $\begin{array}{l}37,834 \\
36,760 \\
32,932 \\
34,380 \\
28,891\end{array}$ & $\begin{array}{l}26,241 \\
25,658 \\
25,329 \\
26,162 \\
26,586\end{array}$ & $\begin{array}{l}19,276 \\
17,444 \\
14,438 \\
16,376 \\
21,187\end{array}$ & $\begin{array}{r}5,575 \\
7,137 \\
10,196 \\
11,888 \\
10,812\end{array}$ & $\begin{array}{l}0 \\
0 \\
0 \\
0 \\
0\end{array}$ & $\begin{array}{l}119,335 \\
118,197 \\
117,676 \\
121,987 \\
124,773\end{array}$ \\
\hline $\begin{array}{l}1985 \\
1986 \\
1987 \\
1988 \\
1989\end{array}$ & $\begin{array}{l}24,255 \\
20,815 \\
25,304 \\
28,698 \\
31,228\end{array}$ & $\begin{array}{l}15,995 \\
12,471 \\
16,385 \\
13,936 \\
17,141\end{array}$ & $\begin{array}{l}25,927 \\
31,911 \\
31,389 \\
39,931 \\
40,963\end{array}$ & $\begin{array}{l}26,956 \\
29,480 \\
27,546 \\
23,994 \\
23,918\end{array}$ & $\begin{array}{l}24,092 \\
22,084 \\
22,926 \\
24,176 \\
22,846\end{array}$ & $\begin{array}{r}10,155 \\
13,204 \\
11,373 \\
9,994 \\
5,539\end{array}$ & $\begin{array}{l}0 \\
0 \\
0 \\
0 \\
0\end{array}$ & $\begin{array}{l}127,380 \\
129,965 \\
134,923 \\
140,729 \\
141,635\end{array}$ \\
\hline $\begin{array}{l}1990 \\
1991 \\
1992 \\
1993 \\
1994\end{array}$ & $\begin{array}{l}30,928 \\
31,584 \\
32,614 \\
29,277 \\
28,399\end{array}$ & $\begin{array}{l}22,483 \\
22,881 \\
29,686 \\
31,761 \\
38,347\end{array}$ & $\begin{array}{l}33,583 \\
27,935 \\
17,928 \\
14,439 \\
10,998\end{array}$ & $\begin{array}{l}27,560 \\
26,257 \\
27,193 \\
28,479 \\
26,800\end{array}$ & $\begin{array}{l}23,624 \\
28,477 \\
24,155 \\
26,889 \\
29,225\end{array}$ & $\begin{array}{r}1,352 \\
6,323 \\
10,167 \\
14,280 \\
11,504\end{array}$ & $\begin{array}{l}1,502 \\
1,622 \\
1,806 \\
1,980 \\
1,972\end{array}$ & $\begin{array}{l}141,032 \\
145,079 \\
143,549 \\
147,105 \\
147,245\end{array}$ \\
\hline
\end{tabular}

1 Includes renewable and indigenous fuels used by non-utility generators.

2 Electricity generated by industrial establishments is excluded.

${ }^{3}$ Generation data are net of station use. 
New York State

Sales of Electricity

by Sector,

1980-1994
Figure 2-6

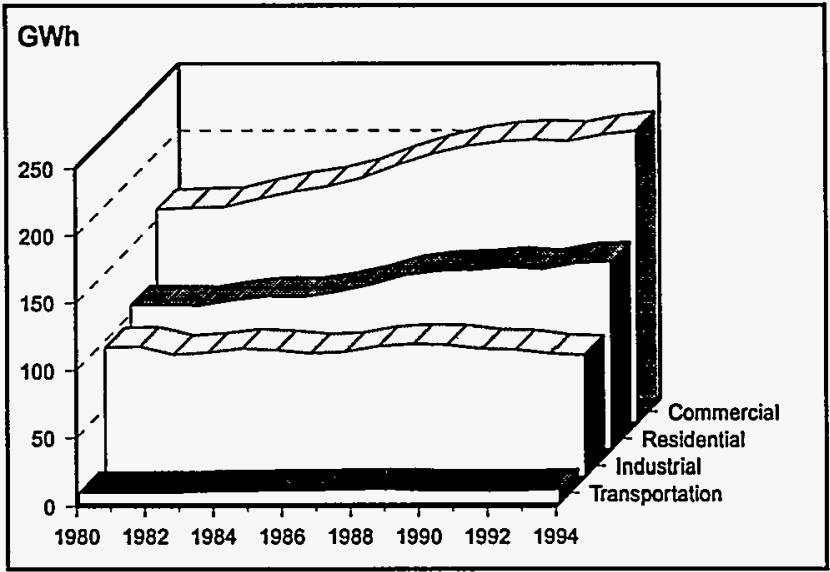

Table 2-6a (Gigawatthours)

\begin{tabular}{|c|c|c|c|c|c|}
\hline Year & $\frac{\text { Residential }}{G W h}$ & $\frac{\text { Commercial }}{\mathrm{GWh}}$ & $\frac{\text { Industrial }}{\mathrm{GWh}}$ & $\frac{\text { Transportation }}{\text { GWh }}$ & $\frac{\text { Total }}{\text { GWh }}$ \\
\hline $\begin{array}{l}1980 \\
1981 \\
1982 \\
1983 \\
1984\end{array}$ & $\begin{array}{l}31,303 \\
31,222 \\
31,157 \\
32,375 \\
33,156\end{array}$ & $\begin{array}{l}46,372 \\
46,813 \\
46,923 \\
48,758 \\
50,286\end{array}$ & $\begin{array}{l}27,745 \\
27,930 \\
26,218 \\
26,662 \\
27,455\end{array}$ & $\begin{array}{l}2,351 \\
2,348 \\
2,350 \\
2,400 \\
2,640\end{array}$ & $\begin{array}{l}107,771 \\
108,313 \\
106,648 \\
110,195 \\
113,537\end{array}$ \\
\hline $\begin{array}{l}1985 \\
1986 \\
1987 \\
1988 \\
1989\end{array}$ & $\begin{array}{l}33,032 \\
34,080 \\
35,696 \\
37,801 \\
38,678\end{array}$ & $\begin{array}{l}51,354 \\
53,204 \\
55,980 \\
58,366 \\
60,166\end{array}$ & $\begin{array}{l}27,121 \\
26,511 \\
26,728 \\
28,087 \\
28,518\end{array}$ & $\begin{array}{l}2,655 \\
2,737 \\
2,855 \\
2,929 \\
3,055\end{array}$ & $\begin{array}{l}114,162 \\
116,532 \\
121,259 \\
127,183 \\
130,417\end{array}$ \\
\hline $\begin{array}{l}1990 \\
1991 \\
1992 \\
1993 \\
1994\end{array}$ & $\begin{array}{l}38,887 \\
39,551 \\
38,962 \\
40,366 \\
40,623\end{array}$ & $\begin{array}{l}61,177 \\
61,665 \\
61,230 \\
62,724 \\
63,594\end{array}$ & $\begin{array}{l}28,350 \\
27,426 \\
27,220 \\
26,341 \\
26,063\end{array}$ & $\begin{array}{l}2,747 \\
2,721 \\
2,679 \\
2,725 \\
2,908\end{array}$ & $\begin{array}{l}131,161 \\
131,363 \\
130,091 \\
132,156 \\
133,188\end{array}$ \\
\hline
\end{tabular}

Table 2-6b (Trillion Btu)

\begin{tabular}{|c|r|r|r|r|r|}
\hline Year & Residential & Commercial & $\frac{\text { Industrial }}{\text { TBtu }}$ & $\frac{\text { Transportation }}{\text { TBtu }}$ & $\frac{\text { Total }}{\text { TBtu }}$ \\
\hline 1980 & TBtu & TBtu & 94.7 & 8.0 & 367.7 \\
1981 & 106.8 & 158.2 & 95.3 & 8.0 & 369.6 \\
1982 & 106.5 & 159.7 & 89.5 & 8.0 & 363.9 \\
1983 & 106.3 & 160.1 & 91.0 & 8.2 & 376.0 \\
1984 & 110.5 & 166.4 & 93.7 & 9.0 & 387.4 \\
\hline 1985 & 113.1 & 171.6 & 92.5 & 9.1 & 389.5 \\
1986 & 112.7 & 175.2 & 90.5 & 9.3 & 397.6 \\
1987 & 116.3 & 181.5 & 91.2 & 10.7 & 413.7 \\
1988 & 121.8 & 191.0 & 95.8 & 10.4 & 433.9 \\
1989 & 129.0 & 199.1 & 97.3 & 945.0 \\
\hline 1990 & 132.0 & 205.3 & 96.4 & 447.5 \\
1991 & 132.7 & 208.7 & 93.6 & 448.2 \\
1992 & 134.9 & 210.4 & 93.6 & 9.3 & 443.9 \\
1993 & 132.9 & 208.9 & 92.9 & 9.1 & 450.9 \\
1994 & 137.7 & 214.0 & 89.9 & 9.3 & 454.4 \\
\hline
\end{tabular}


New York State

Net Consumption

by Sector,

1980-1994
Figure 2-7

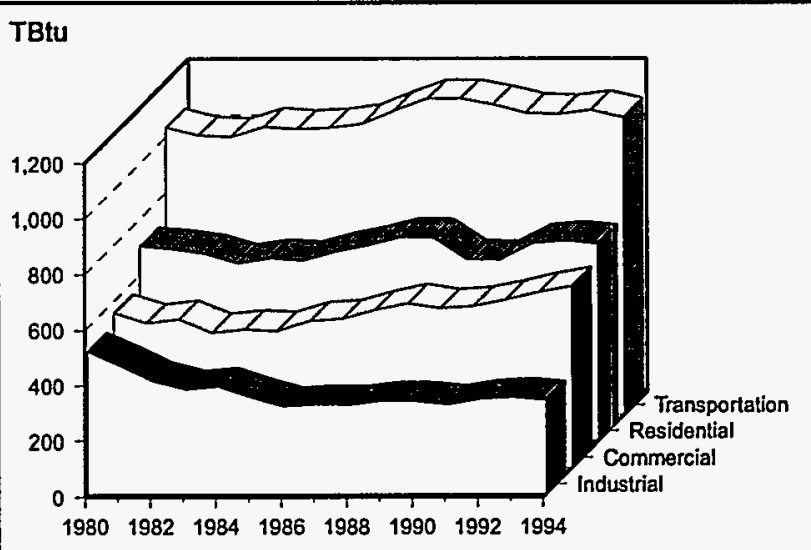

Table 2-7 (Trillion Btu)

\begin{tabular}{|c|c|c|c|c|c|c|c|}
\hline Year & $\frac{\text { Ressidential }}{\text { TBtu }}$ & $\frac{\text { Commercial }}{\text { TBtu }}$ & $\frac{\text { Industrial }}{\text { TBtu }}$ & $\frac{\text { Transportation }}{\text { TBtu }}$ & $\begin{array}{r}\begin{array}{r}\text { Net } \\
\text { Consumption }\end{array} \\
\text { TBtu }\end{array}$ & $\begin{array}{r}\text { Electric } \\
\text { Generation } \\
\text { Losses }^{1} \\
\text { TBtu }\end{array}$ & $\frac{\begin{array}{r}\text { Primary } \\
\text { Consumption }\end{array}}{\text { TBtu }}$ \\
\hline $\begin{array}{l}1980 \\
1981 \\
1982 \\
1983 \\
1984\end{array}$ & $\begin{array}{l}701.4 \\
691.4 \\
674.5 \\
639.1 \\
657.6\end{array}$ & $\begin{array}{l}556.5 \\
519.9 \\
533.7 \\
485.8 \\
498.5\end{array}$ & $\begin{array}{l}512.7 \\
459.7 \\
403.8 \\
374.3 \\
385.3\end{array}$ & $\begin{array}{l}1,035.8 \\
1,006.0 \\
1,001.2 \\
1,036.2 \\
1,028.8\end{array}$ & $\begin{array}{l}2,806.4 \\
2,677.0 \\
2,613.2 \\
2,535.4 \\
2,570.2\end{array}$ & $\begin{array}{l}943.0 \\
924.4 \\
908.9 \\
931.2 \\
962.3\end{array}$ & $\begin{array}{l}3,749.4 \\
3,601.4 \\
3,522.1 \\
3,466.7 \\
3,532.6\end{array}$ \\
\hline $\begin{array}{l}1985 \\
1986 \\
1987 \\
1988 \\
1989\end{array}$ & $\begin{array}{l}650.6 \\
681.2 \\
703.6 \\
734.5 \\
731.9\end{array}$ & $\begin{array}{l}491.2 \\
528.1 \\
535.0 \\
568.0 \\
591.0\end{array}$ & $\begin{array}{l}345.7 \\
312.8 \\
321.1 \\
318.4 \\
334.6\end{array}$ & $\begin{array}{l}1,033.6 \\
1,049.4 \\
1,096.8 \\
1,139.2 \\
1,138.8\end{array}$ & $\begin{array}{l}2,521.1 \\
2,571.4 \\
2,656.5 \\
2,760.2 \\
2,796.4\end{array}$ & $\begin{array}{r}968.0 \\
974.6 \\
998.0 \\
1,018.0 \\
1,035.2\end{array}$ & $\begin{array}{l}3,489.1 \\
3,546.0 \\
3,654.5 \\
3,778.2 \\
3,831.5\end{array}$ \\
\hline $\begin{array}{l}1990 \\
1991 \\
1992 \\
1993 \\
1994\end{array}$ & $\begin{array}{l}654.4 \\
651.0 \\
710.8 \\
720.6 \\
709.1\end{array}$ & $\begin{array}{l}572.5 \\
581.0 \\
604.8 \\
631.4 \\
650.9\end{array}$ & $\begin{array}{l}331.6 \\
320.5 \\
339.9 \\
345.7 \\
335.4\end{array}$ & $\begin{array}{l}1,118.4 \\
1,088.3 \\
1,083.8 \\
1,097.7 \\
1,071.7\end{array}$ & $\begin{array}{l}2,676.9 \\
2,640.8 \\
2,739.4 \\
2,795.3 \\
2,767.1\end{array}$ & $\begin{array}{l}1,026.4 \\
1,066.9 \\
1,075.8 \\
1,117.2 \\
1,112.7\end{array}$ & $\begin{array}{l}3,703.3 \\
3,707.7 \\
3,815.2 \\
3,912.6 \\
3,879,7\end{array}$ \\
\hline
\end{tabular}

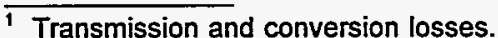


New York State

Net Residential Consumption

by Fuel Type,

1980-1994
Figure 2-8

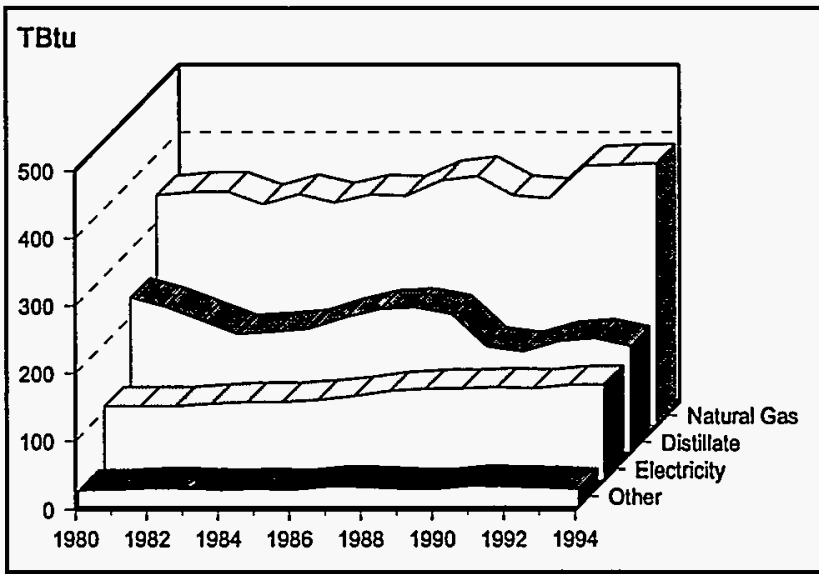

Table 2-8a (Physical Units)

\begin{tabular}{|r|r|r|r|r|r|r|r|}
\hline Year & $\begin{array}{r}\text { Coal' } \\
\text { MTons }\end{array}$ & $\begin{array}{r}\text { Natural } \\
\text { Gas } \\
\text { MMcf }\end{array}$ & $\frac{\text { Distillate }}{\text { Mbbl }}$ & $\frac{\text { Kerosene }}{\text { Mbbl }}$ & $\frac{\text { LPG }}{\text { Mbbl }}$ & $\begin{array}{r}\text { Total } \\
\text { Petroleum } \\
\text { Mbbl }\end{array}$ & $\begin{array}{r}\text { Electricity } \\
\text { GWh }\end{array}$ \\
\hline 1980 & 181 & 333,382 & 39,397 & 1,755 & 2,511 & 43,663 & 31,303 \\
1981 & 235 & 337,479 & 36,851 & 1,528 & 2,668 & 41,047 & 31,222 \\
1982 & 286 & 338,112 & 33,383 & 1,859 & 2,501 & 37,743 & 31,157 \\
1983 & 180 & 318,309 & 29,926 & 1,849 & 2,974 & 34,749 & 32,375 \\
1984 & 224 & 332,991 & 30,503 & 1,253 & 2,963 & 34,719 & 33,156 \\
\hline 1985 & 208 & 320,385 & 31,280 & 1,459 & 3,227 & 35,966 & 33,032 \\
1986 & 206 & 332,584 & 34,140 & 1,120 & 3,282 & 38,542 & 34,080 \\
1987 & 173 & 330,508 & 36,319 & 1,840 & 3,834 & 41,993 & 35,696 \\
1988 & 139 & 352,829 & 36,717 & 1,923 & 3,718 & 42,358 & 37,801 \\
1989 & 137 & 358,561 & 35,030 & 1,354 & 3,931 & 40,315 & 38,678 \\
\hline 1990 & 123 & 331,157 & 26,679 & 1,164 & 4,079 & 31,922 & 38,887 \\
1991 & 128 & 326,974 & 25,512 & 1,389 & 5,051 & 31,952 & 39,551 \\
1992 & 135 & 372,862 & 28,165 & 1,252 & 4,965 & 34,382 & 38,962 \\
1993 & 116 & 375,093 & 28,846 & 1,565 & 4,293 & 34,704 & 40,366 \\
1994 & 85 & 376,738 & 26,905 & 1,396 & 4,005 & 32,306 & 40,623 \\
\hline
\end{tabular}

Table 2-8b (Trillion Btu)

\begin{tabular}{|c|r|r|r|r|r|r|r|r|}
\hline Year & $\frac{\text { Coal' }}{\text { TBtu }}$ & $\begin{array}{r}\text { Natural } \\
\text { Gas } \\
\text { TBtu }\end{array}$ & $\frac{\text { Distillate }}{\text { TBtu }}$ & $\frac{\text { Kerosene }}{\text { TBtu }}$ & $\frac{\text { LPG }}{\text { TBtu }}$ & $\begin{array}{r}\text { Total } \\
\text { Petroleum } \\
\text { TBtu }\end{array}$ & $\begin{array}{r}\text { Electricity } \\
\text { TBtu }\end{array}$ & $\frac{\text { Total }}{\text { TBtu }}$ \\
\hline 1980 & 4.1 & 341.4 & 229.5 & 10.0 & 9.6 & 249.1 & 106.8 & 701.4 \\
1981 & 5.4 & 345.9 & 214.7 & 8.7 & 10.2 & 233.6 & 106.5 & 691.4 \\
1982 & 6.7 & 346.9 & 194.5 & 10.5 & 9.6 & 214.6 & 106.3 & 674.5 \\
1983 & 4.3 & 328.2 & 174.3 & 10.5 & 11.4 & 196.2 & 110.5 & 639.1 \\
1984 & 5.4 & 343.0 & 177.7 & 7.1 & 11.4 & 196.2 & 113.1 & 657.6 \\
\hline 1985 & 4.8 & 330.3 & 182.2 & 8.3 & 12.4 & 202.9 & 112.7 & 650.6 \\
1986 & 4.8 & 342.2 & 198.9 & 6.4 & 12.6 & 217.8 & 116.3 & 681.2 \\
1987 & 4.3 & 340.8 & 211.6 & 10.4 & 14.7 & 236.7 & 121.8 & 703.6 \\
1988 & 3.4 & 363.1 & 213.9 & 10.9 & 14.3 & 239.0 & 129.0 & 734.5 \\
1989 & 3.5 & 369.7 & 204.0 & 7.7 & 15.1 & 226.8 & 132.0 & 731.9 \\
\hline 1990 & 2.9 & 341.1 & 155.4 & 6.6 & 15.6 & 177.7 & 132.7 & 654.4 \\
1991 & 3.1 & 337.1 & 148.6 & 7.9 & 19.4 & 175.9 & 134.9 & 651.0 \\
1992 & 3.2 & 384.4 & 164.1 & 7.1 & 19.0 & 190.2 & 132.9 & 710.8 \\
1993 & 2.8 & 386.7 & 168.0 & 8.9 & 16.5 & 193.4 & 137.7 & 720.6 \\
1994 & 2.1 & 388.4 & 156.7 & 7.9 & 15.4 & 180.0 & 138.6 & 709.1 \\
\hline
\end{tabular}

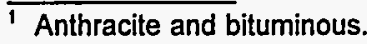


New York State

Net Commercial Consumption by Fuel Type, 1980-1994
Figure 2-9

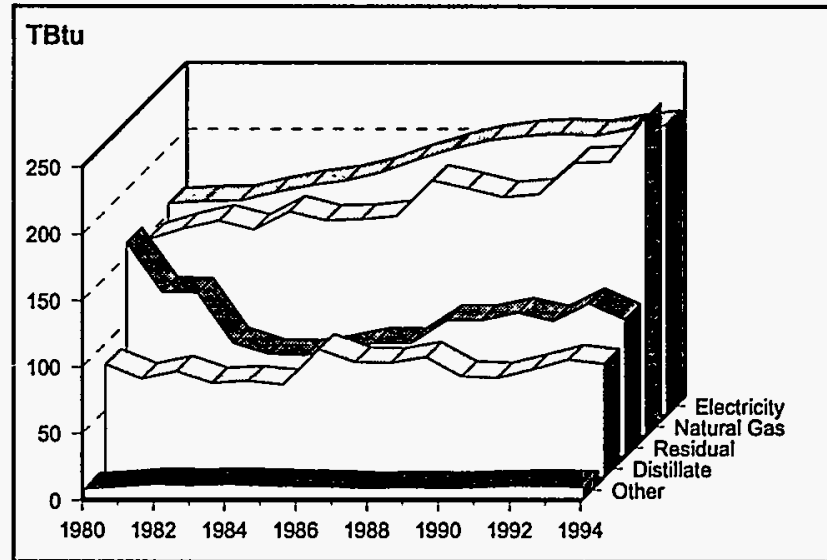

Table 2-9a (Physical Units)

\begin{tabular}{|r|r|r|r|r|r|r|r|r|}
\hline Year & $\begin{array}{r}\text { Cogal } \\
\text { MTons }\end{array}$ & $\begin{array}{r}\text { Natural } \\
\text { Gas } \\
\text { MMcf }\end{array}$ & $\frac{\text { Distillate }}{\text { Mbbl }}$ & $\frac{\text { Residual }}{\text { Mbbl }}$ & $\frac{\text { Kerosene }}{\text { Mbbl }}$ & $\frac{\text { LPG }}{\text { Mbbl }}$ & $\begin{array}{r}\text { Total } \\
\text { Petroleum } \\
\text { Mbbl }\end{array}$ & $\begin{array}{r}\text { Electricity } \\
\text { GWh }\end{array}$ \\
\hline 1980 & 176 & 143,494 & 14,518 & 25,470 & 169 & 443 & 40,600 & 46,372 \\
1981 & 249 & 151,363 & 12,628 & 19,577 & 158 & 471 & 32,834 & 46,813 \\
1982 & 335 & 157,064 & 13,638 & 19,482 & 199 & 441 & 33,760 & 46,923 \\
1983 & 210 & 149,782 & 12,119 & 13,507 & 469 & 525 & 26,620 & 48,758 \\
1984 & 265 & 163,125 & 12,352 & 12,185 & 374 & 523 & 25,434 & 50,286 \\
\hline 1985 & 226 & 156,457 & 11,891 & 12,073 & 391 & 569 & 24,924 & 51,354 \\
1986 & 258 & 157,370 & 16,492 & 12,672 & 116 & 579 & 29,859 & 53,204 \\
1987 & 198 & 159,626 & 14,784 & 13,509 & 182 & 677 & 29,152 & 55,980 \\
1988 & 170 & 185,939 & 14,719 & 13,471 & 96 & 656 & 28,942 & 58,366 \\
1989 & 161 & 179,759 & 15,470 & 16,271 & 254 & 694 & 32,689 & 60,166 \\
\hline 1990 & 150 & 173,560 & 12,942 & 16,276 & 177 & 720 & 30,115 & 61,177 \\
1991 & 151 & 175,372 & 12,753 & 17,132 & 141 & 891 & 30,917 & 61,665 \\
1992 & 141 & 198,189 & 13,904 & 16,156 & 408 & 876 & 31,344 & 61,230 \\
1993 & 116 & 199,232 & 15,112 & 18,262 & 616 & 758 & 34,748 & 62,724 \\
1994 & 101 & 230,664 & 14,571 & 16,376 & 538 & 731 & 32,216 & 63,594 \\
\hline
\end{tabular}

Table 2-9b (Trillion Btu)

\begin{tabular}{|c|c|c|c|c|c|c|c|c|c|}
\hline Year & $\frac{\text { Coal }}{\text { TBtu }}$ & $\begin{array}{r}\text { Natural } \\
\text { Gas } \\
\text { TBtu }\end{array}$ & $\frac{\text { Distillate }}{\text { TBtu }}$ & $\frac{\text { Residual }}{\text { TBtu }}$ & $\frac{\text { Kerosene }}{\text { TBtu }}$ & $\frac{\mathrm{LPG}}{\mathrm{TBtu}}$ & $\begin{array}{r}\begin{array}{r}\text { Total } \\
\text { Petroleum }\end{array} \\
\text { TBtu }\end{array}$ & $\frac{\text { Electricity }}{\text { TBtu }}$ & $\frac{\text { Total }}{\text { TBtu }}$ \\
\hline 1980 & 4.0 & 146.9 & 84.6 & 160.1 & 1.0 & 1.7 & 247.4 & 158.2 & 556.5 \\
\hline 1981 & 5.7 & 155.1 & 73.6 & 123.1 & 0.9 & 1.8 & 199.3 & 159.7 & 519.9 \\
\hline 1982 & 7.7 & 161.1 & 79.4 & 122.5 & 1.1 & 1.7 & 204.7 & 160.1 & 533.7 \\
\hline 1983 & 4.9 & 154.4 & 70.6 & 84.9 & 2.7 & 2.0 & 160.2 & 166.4 & 485.8 \\
\hline 1984 & 6.2 & 168.0 & 72.0 & 76.6 & 2.1 & 2.0 & 152.7 & 171.6 & 498.5 \\
\hline 1985 & 5.1 & 161.3 & 69.3 & 75.9 & 2.2 & 2.2 & 149.6 & 175.2 & 491.2 \\
\hline 1986 & 6.0 & 161.9 & 96.1 & 79.7 & 0.7 & 2.2 & 178.6 & 181.5 & 528.1 \\
\hline 1987 & 4.8 & 164.6 & 86.1 & 84.9 & 1.0 & 2.6 & 174.7 & 191.0 & 535.0 \\
\hline 1988 & 4.1 & 191.3 & 85.7 & 84.7 & 0.5 & 2.5 & 173.5 & 199.1 & 568.0 \\
\hline 1989 & 3.9 & 185.3 & 90.1 & 102.3 & 1.4 & 2.7 & 196.5 & 205.3 & 591.0 \\
\hline 1990 & 3.5 & 178.8 & 75.4 & 102.3 & 1.0 & 2.8 & 181.5 & 208.7 & 572.5 \\
\hline 1991 & 3.5 & 180.8 & 74.3 & 107.7 & 0.8 & 3.4 & 186.2 & 210.4 & 581.0 \\
\hline 1992 & 3.3 & 204.3 & 81.0 & 101.6 & 2.3 & 3.4 & 188.2 & 208.9 & 604.8 \\
\hline 1993 & 2.7 & 205.4 & 88.0 & 114.8 & 3.5 & 2.9 & 209.2 & 214.0 & 631.4 \\
\hline 1994 & 2.4 & 237.8 & 84.9 & 103.0 & 3.1 & 2.8 & 193.7 & 217.0 & 650.9 \\
\hline
\end{tabular}

Anthracite and bituminous. 
New York State

Net Industrial Consumption

by Fuel Type,

1980-1994
Figure 2-10

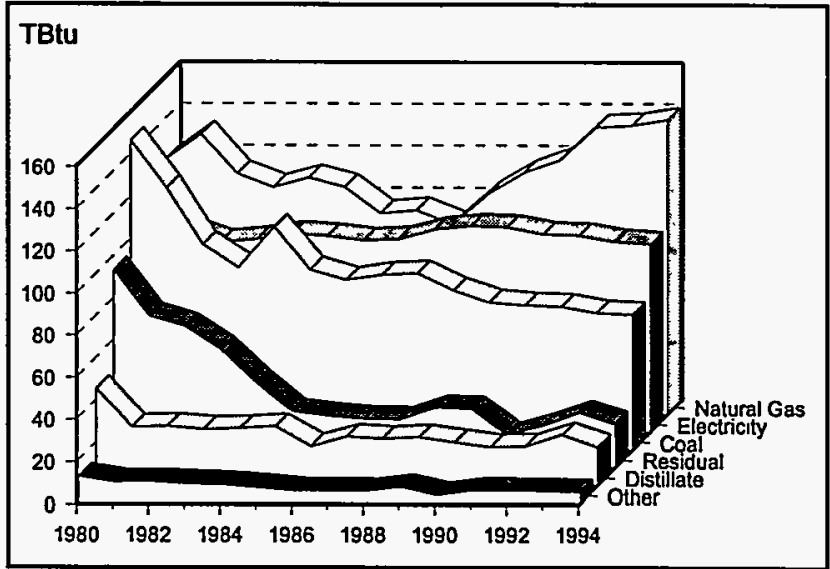

Table 2-10a (Physical Units)

\begin{tabular}{|r|r|r|r|r|r|r|r|r|}
\hline Year & $\begin{array}{r}\text { Coal }^{12} \\
\text { MTons }\end{array}$ & $\begin{array}{r}\text { Natural } \\
\text { Gas }^{3} \\
\text { MMcf }\end{array}$ & $\frac{\text { Distillate }}{\text { Mbbl }}$ & $\frac{\text { Residual }}{\text { Mbbl }}$ & $\frac{\text { Kerosene }}{\text { Mbbl }}$ & $\frac{1}{\text { LbG }}$ & $\begin{array}{r}\text { Total } \\
\text { Petroleum } \\
\text { Mbbl }\end{array}$ & $\begin{array}{r}\text { Electricity } \\
\text { GWh }\end{array}$ \\
\hline 1980 & 5,886 & 117,879 & 7,790 & 14,836 & 385 & 2,598 & 25,609 & 27,745 \\
1981 & 4,993 & 129,458 & 4,650 & 11,362 & 386 & 1,917 & 18,315 & 27,930 \\
1982 & 3,954 & 110,963 & 4,726 & 10,567 & 503 & 1,795 & 17,591 & 26,218 \\
1983 & 3,590 & 104,086 & 4,451 & 8,800 & 689 & 1,240 & 15,180 & 26,662 \\
1984 & 4,373 & 108,750 & 4,537 & 6,316 & 522 & 1,359 & 12,734 & 27,455 \\
\hline 1985 & 3,609 & 104,304 & 4,723 & 4,138 & 561 & 980 & 10,402 & 27,121 \\
1986 & 3,308 & 92,423 & 3,070 & 3,810 & 316 & 909 & 8,105 & 26,511 \\
1987 & 3,385 & 93,749 & 3,855 & 3,615 & 360 & 878 & 8,708 & 26,728 \\
1988 & 3,387 & 87,501 & 3,698 & 3,581 & 412 & 742 & 8,433 & 28,087 \\
1989 & 3,127 & 101,114 & 3,845 & 4,474 & 736 & 801 & 9,856 & 28,518 \\
\hline 1990 & 2,899 & 110,964 & 3,419 & 4,381 & 163 & 655 & 8,618 & 28,350 \\
1991 & 2,847 & 116,915 & 3,042 & 2,387 & 223 & 1,107 & 6,759 & 27,426 \\
1992 & 2,813 & 132,126 & 3,117 & 3,125 & 201 & 1,092 & 7,535 & 27,220 \\
1993 & 2,686 & 132,821 & 4,045 & 4,073 & 240 & 962 & 9,320 & 26,341 \\
1994 & 2,641 & 135,469 & 3,062 & 3,220 & 355 & 728 & 7,365 & 26,063 \\
\hline
\end{tabular}

Table 2-10b (Trillion Btu)

\begin{tabular}{|c|c|c|c|c|c|c|c|c|c|}
\hline Year & $\frac{\text { Coall }^{1,2}}{\text { TBtu }^{1}}$ & $\begin{array}{c}\text { Natural } \\
\frac{\text { Gas }^{3}}{\text { TBtu }}\end{array}$ & $\frac{\text { Distillate }}{\text { TBtu }}$ & $\frac{\text { Residual }}{\text { TBtu }}$ & $\frac{\text { Kerosene }}{\text { TBtu }}$ & $\frac{\text { LPG }}{\text { TBtu }}$ & $\begin{array}{r}\begin{array}{r}\text { Total } \\
\text { Petroleum }\end{array} \\
\text { TBtu }\end{array}$ & $\frac{\text { Electricity }}{\text { TBtu }}$ & $\frac{\text { Total }^{4,5}}{\text { TBtu }^{4}}$ \\
\hline $\begin{array}{l}1980 \\
1981 \\
1982 \\
1983 \\
1984\end{array}$ & $\begin{array}{r}146.5 \\
123.6 \\
96.8 \\
86.1 \\
105.3\end{array}$ & $\begin{array}{l}120.7 \\
132.7 \\
113.8 \\
107.3 \\
112.0\end{array}$ & $\begin{array}{l}45.4 \\
27.1 \\
27.5 \\
25.9 \\
26.4\end{array}$ & $\begin{array}{l}93.3 \\
71.4 \\
66.4 \\
55.3 \\
39.7\end{array}$ & $\begin{array}{l}2.2 \\
2.2 \\
2.9 \\
3.9 \\
3.0\end{array}$ & $\begin{array}{r}10.0 \\
7.4 \\
6.9 \\
4.8 \\
5.2\end{array}$ & $\begin{array}{r}150.8 \\
108.1 \\
103.7 \\
89.9 \\
74.3\end{array}$ & $\begin{array}{l}94.7 \\
95.3 \\
89.5 \\
91.0 \\
93.7\end{array}$ & $\begin{array}{l}512.7 \\
459.7 \\
403.8 \\
374.3 \\
385.3\end{array}$ \\
\hline $\begin{array}{l}1985 \\
1986 \\
1987 \\
1988 \\
1989\end{array}$ & $\begin{array}{l}85.1 \\
80.2 \\
82.7 \\
83.3 \\
75.3\end{array}$ & $\begin{array}{r}107.5 \\
95.1 \\
96.7 \\
90.0 \\
104.2\end{array}$ & $\begin{array}{l}27.5 \\
17.9 \\
22.5 \\
21.5 \\
22.4\end{array}$ & $\begin{array}{l}26.0 \\
24.0 \\
22.7 \\
22.5 \\
28.1\end{array}$ & $\begin{array}{l}3.2 \\
1.8 \\
2.0 \\
2.3 \\
4.2\end{array}$ & $\begin{array}{l}3.8 \\
3.5 \\
3.4 \\
2.8 \\
3.1\end{array}$ & $\begin{array}{l}60.5 \\
47.1 \\
50.6 \\
49.2 \\
57.8\end{array}$ & $\begin{array}{l}92.5 \\
90.5 \\
91.2 \\
95.8 \\
97.3\end{array}$ & $\begin{array}{l}345.7 \\
312.8 \\
321.1 \\
318.4 \\
334.6\end{array}$ \\
\hline $\begin{array}{l}1990 \\
1991 \\
1992 \\
1993 \\
1994\end{array}$ & $\begin{array}{l}69.7 \\
68.2 \\
67.7 \\
64.6 \\
63.9\end{array}$ & $\begin{array}{l}114.3 \\
120.5 \\
136.2 \\
136.9 \\
139.7\end{array}$ & $\begin{array}{l}19.9 \\
17.7 \\
18.2 \\
23.6 \\
17.8\end{array}$ & $\begin{array}{l}27.5 \\
15.0 \\
19.6 \\
25.6 \\
20.2\end{array}$ & $\begin{array}{l}0.9 \\
1.3 \\
1.1 \\
1.4 \\
2.0\end{array}$ & $\begin{array}{l}2.5 \\
4.2 \\
4.2 \\
3.7 \\
2.8\end{array}$ & $\begin{array}{l}50.9 \\
38.2 \\
43.1 \\
54.2 \\
42.9\end{array}$ & $\begin{array}{l}96.7 \\
93.6 \\
92.9 \\
89.9 \\
88.9\end{array}$ & $\begin{array}{l}331.6 \\
320.5 \\
339.9 \\
345.7 \\
335.4\end{array}$ \\
\hline
\end{tabular}

Anthracite and bituminous.

2 Includes deliveries to cokeries.

3 Excludes lease and plant fuels.

4 Excludes non-fuel uses, e.g., feedstock.

5 Includes those fuels used by industry to generate electricity and process steam. 
New York State

Net Transportation Consumption by Fuel Type,

1980-1994
Figure 2-11

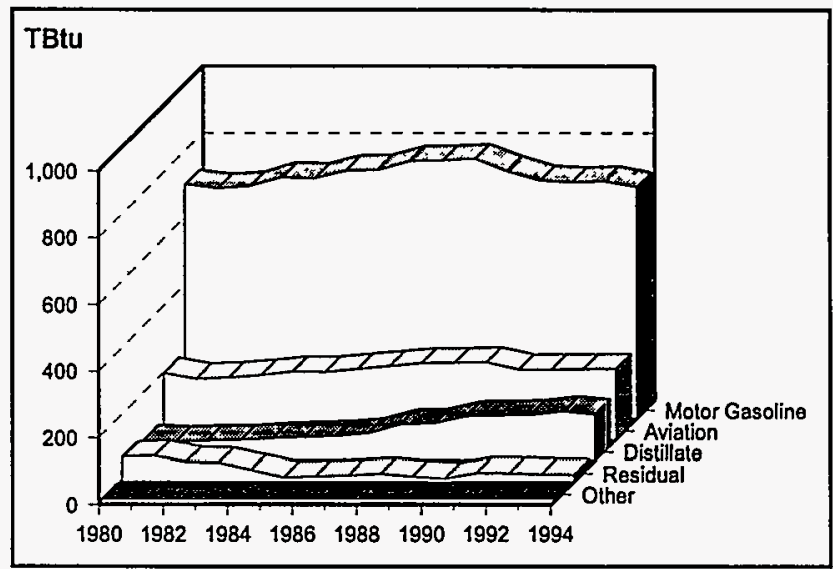

Table 2-11a (Physical Units)

\begin{tabular}{|c|c|c|c|c|c|c|c|}
\hline Year & $\frac{\text { Distillate }}{\text { Mbbl }}$ & $\frac{\text { Residual }}{\mathrm{Mbbl}}$ & $\begin{array}{r}\begin{array}{r}\text { Motor } \\
\text { Gasoline }\end{array} \\
\text { Mbbl }\end{array}$ & $\begin{array}{l}\text { Aviation } \\
\frac{\text { Fuels' }}{\text { Mbbl }}\end{array}$ & $\frac{\mathrm{LPG}}{\mathrm{Mbbl}}$ & $\begin{array}{r}\begin{array}{r}\text { Total } \\
\text { Petroleum }\end{array} \\
\text { Mbbl }\end{array}$ & $\frac{\text { Electricity }}{G W h}$ \\
\hline $\begin{array}{l}1980 \\
1981 \\
1982 \\
1983 \\
1984\end{array}$ & $\begin{array}{r}10,050 \\
9,237 \\
9,907 \\
10,207 \\
11,208\end{array}$ & $\begin{array}{r}11,367 \\
11,854 \\
8,997 \\
8,600 \\
5,263\end{array}$ & $\begin{array}{l}133,282 \\
131,037 \\
132,218 \\
137,445 \\
136,788\end{array}$ & $\begin{array}{l}34,808 \\
31,872 \\
32,416 \\
33,838 \\
35,644\end{array}$ & $\begin{array}{r}79 \\
159 \\
140 \\
167 \\
210\end{array}$ & $\begin{array}{l}189,586 \\
184,159 \\
183,678 \\
190,257 \\
189,113\end{array}$ & $\begin{array}{l}2,351 \\
2,348 \\
2,350 \\
2,400 \\
2,640\end{array}$ \\
\hline $\begin{array}{l}1985 \\
1986 \\
1987 \\
1988 \\
1989\end{array}$ & $\begin{array}{l}11,882 \\
12,222 \\
13,626 \\
18,197 \\
18,675\end{array}$ & $\begin{array}{l}1,766 \\
2,191 \\
2,783 \\
3,621 \\
2,190\end{array}$ & $\begin{array}{l}141,249 \\
141,699 \\
146,758 \\
147,000 \\
147,800\end{array}$ & $\begin{array}{l}35,573 \\
37,090 \\
38,607 \\
40,175 \\
40,360\end{array}$ & $\begin{array}{r}147 \\
108 \\
87 \\
122 \\
153\end{array}$ & $\begin{array}{l}190,617 \\
193,310 \\
201,861 \\
209,115 \\
209,178\end{array}$ & $\begin{array}{l}2,655 \\
2,737 \\
2,855 \\
2,929 \\
3,055\end{array}$ \\
\hline $\begin{array}{l}1990 \\
1991 \\
1992 \\
1993 \\
1994\end{array}$ & $\begin{array}{l}22,310 \\
22,729 \\
23,066 \\
25,076 \\
23,481\end{array}$ & $\begin{array}{l}1,224 \\
3,979 \\
3,767 \\
3,394 \\
3,184\end{array}$ & $\begin{array}{l}140,900 \\
135,660 \\
134,600 \\
135,350 \\
131,988\end{array}$ & $\begin{array}{l}40,680 \\
36,750 \\
36,870 \\
36,950 \\
37,250\end{array}$ & $\begin{array}{l}152 \\
158 \\
144 \\
126 \\
118\end{array}$ & $\begin{array}{l}205,266 \\
199,276 \\
198,447 \\
200,896 \\
196,021\end{array}$ & $\begin{array}{l}2,747 \\
2,721 \\
2,679 \\
2,725 \\
2,908\end{array}$ \\
\hline
\end{tabular}

Table 2-11b (Trillion Btu)

\begin{tabular}{|c|c|c|c|c|c|c|c|c|}
\hline Year & $\frac{\text { Distillate }}{\text { TBtu }}$ & $\frac{\text { Residual }}{\text { TBtu }}$ & $\begin{array}{r}\text { Motor } \\
\text { Gassoline } \\
\text { TBtu }\end{array}$ & $\begin{array}{l}\text { Aviation } \\
\text { Fuels' } \\
\text { TBtu }\end{array}$ & $\frac{\text { LPG }}{\text { TBtu }}$ & $\begin{array}{r}\text { Total } \\
\text { Petroleum } \\
\text { TBtu }\end{array}$ & $\frac{\text { Electricity }}{\text { TBtu }}$ & $\frac{\text { Total }^{2}}{\text { TBtu }}$ \\
\hline $\begin{array}{l}1980 \\
1981 \\
1982 \\
1983 \\
1984\end{array}$ & $\begin{array}{l}58.5 \\
53.8 \\
57.7 \\
59.5 \\
65.3\end{array}$ & $\begin{array}{l}71.5 \\
74.5 \\
56.6 \\
54.1 \\
33.1\end{array}$ & $\begin{array}{l}700.1 \\
688.3 \\
694.5 \\
722.0 \\
718.5\end{array}$ & $\begin{array}{l}197.4 \\
180.7 \\
183.8 \\
191.9 \\
202.1\end{array}$ & $\begin{array}{l}0.3 \\
0.6 \\
0.5 \\
0.6 \\
0.8\end{array}$ & $\begin{array}{r}1,027.8 \\
998.0 \\
993.1 \\
1,028.0 \\
1,019.8\end{array}$ & $\begin{array}{l}8.0 \\
8.0 \\
8.0 \\
8.2 \\
9.0\end{array}$ & $\begin{array}{l}1,035.8 \\
1,006.0 \\
1,001.2 \\
1,036.2 \\
1,028.8\end{array}$ \\
\hline $\begin{array}{l}1985 \\
1986 \\
1987 \\
1988 \\
1989\end{array}$ & $\begin{array}{r}69.2 \\
71.2 \\
79.4 \\
106.0 \\
108.8\end{array}$ & $\begin{array}{l}11.1 \\
13.8 \\
17.5 \\
22.8 \\
13.8\end{array}$ & $\begin{array}{l}742.0 \\
744.3 \\
770.9 \\
772.2 \\
776.4\end{array}$ & $\begin{array}{l}201.7 \\
210.3 \\
218.9 \\
227.8 \\
228.8\end{array}$ & $\begin{array}{l}0.6 \\
0.4 \\
0.3 \\
0.5 \\
0.6\end{array}$ & $\begin{array}{l}1,024.6 \\
1,040.0 \\
1,087.0 \\
1,129.2 \\
1,128.4\end{array}$ & $\begin{array}{r}9.1 \\
9.3 \\
9.7 \\
10.0 \\
10.4\end{array}$ & $\begin{array}{l}1,033.6 \\
1,049.4 \\
1,096.8 \\
1,139.2 \\
1,138.8\end{array}$ \\
\hline $\begin{array}{l}1990 \\
1991 \\
1992 \\
1993 \\
1994\end{array}$ & $\begin{array}{l}130.0 \\
132.4 \\
134.4 \\
146.1 \\
136.8\end{array}$ & $\begin{array}{r}7.7 \\
25.0 \\
23.7 \\
21.3 \\
20.0\end{array}$ & $\begin{array}{l}740.1 \\
712.6 \\
707.1 \\
711.0 \\
693.3\end{array}$ & $\begin{array}{l}230.7 \\
208.4 \\
209.1 \\
209.5 \\
211.2\end{array}$ & $\begin{array}{l}0.6 \\
0.6 \\
0.6 \\
0.5 \\
0.5\end{array}$ & $\begin{array}{l}1,109.0 \\
1,079.0 \\
1,074.7 \\
1,088.4 \\
1,061.8\end{array}$ & $\begin{array}{l}9.4 \\
9.3 \\
9.1 \\
9.3 \\
9.9\end{array}$ & $\begin{array}{l}1,118.4 \\
1,088.3 \\
1,083.8 \\
1,097.7 \\
1,071.7\end{array}$ \\
\hline
\end{tabular}

\footnotetext{
1 Aviation gasoline and kerosene-type jet fuel.

2 Excludes pipeline fuels.
} 


\section{Section 3}

\section{NEW YORK STATE ENERGY PRICES}

This section presents data on energy prices at the retail level for the period 1980 though 1994. Retail energy prices are provided by fuel type for the residential, commercial, industrial, and transportation sectors in nominal dollars cost per physical unit and per million Btu.

Historical petroleum prices have been compiled from U.S. DOE/EIA's State Energy Price and Expenditure Report. For those years in which the former New York State Energy Office conducted surveys of various fuels, their results are provided.

Historical coal and natural gas data also have been compiled from U.S. DOE/EIA's State Energy Price and Expenditure Report. In the case of electricity, however, prices for all sectors were developed from data provided on U.S. DOE/EIA FORM-826, Monthly Electric Utility Sales and Revenue Report with State Distributions. 
New York State

Residential Energy Prices, 1980-1994
Figure 3-1

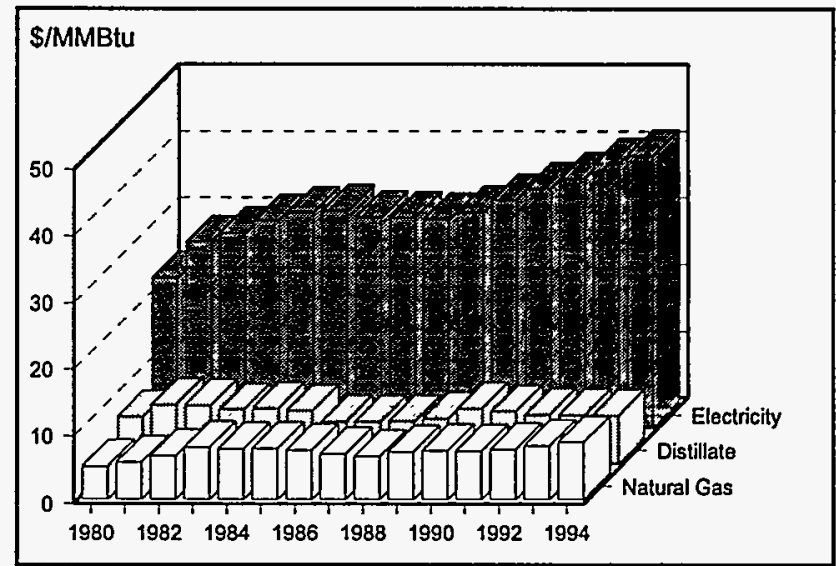

Table 3-1a (Physical Units)

\begin{tabular}{|r|r|r|r|r|r|r|}
\hline Year & $\begin{array}{r}\text { Coal }^{1} \\
\$ / \text { Ton }^{1}\end{array}$ & $\begin{array}{r}\text { Distillate } \\
\text { Cents/Gal }\end{array}$ & $\begin{array}{r}\text { Kerosene } \\
\text { Cents/Gal }\end{array}$ & $\begin{array}{r}\text { LPG }^{3} \\
\text { Cents/Gal }\end{array}$ & $\begin{array}{r}\text { Natural } \\
\text { Gas } \\
\$ / \text { Mcf }\end{array}$ & $\begin{array}{r}\text { Electricity } \\
\text { Cents/kWh }\end{array}$ \\
\hline 1980 & 98.00 & 97.80 & 103.25 & 83.30 & 4.96 & 7.72 \\
1981 & 117.00 & 122.83 & 133.00 & 89.99 & 5.54 & 9.56 \\
1982 & 132.00 & 121.12 & 130.75 & 93.46 & 6.55 & 10.02 \\
1983 & 128.00 & 112.65 & 124.25 & 96.36 & 7.91 & 10.65 \\
1984 & 129.00 & 114.53 & 125.25 & 91.61 & 7.67 & 11.05 \\
\hline 1985 & 134.00 & 110.03 & 119.50 & 101.55 & 7.77 & 11.15 \\
1986 & 138.00 & 87.64 & 103.25 & 90.94 & 7.47 & 10.83 \\
1987 & 138.00 & 88.00 & 96.25 & 88.05 & 6.89 & 10.79 \\
1988 & 142.00 & 88.39 & 100.25 & 87.06 & 6.50 & 10.73 \\
1989 & 145.00 & 93.94 & 102.50 & 105.40 & 7.23 & 11.08 \\
\hline 1990 & 146.00 & 114.82 & 131.75 & 124.53 & 7.41 & 11.71 \\
1991 & 144.00 & 109.18 & 123.50 & 133.26 & 7.38 & 12.31 \\
1992 & 142.00 & 102.90 & 113.50 & 131.83 & 7.60 & 12.82 \\
1993 & 144.00 & 101.72 & 112.75 & 135.13 & 8.15 & 13.55 \\
1994 & 148.00 & 99.85 & 112.00 & 133.37 & 8.73 & 13.98 \\
\hline
\end{tabular}

Table 3-1b (\$/Million Btu)

\begin{tabular}{|c|c|c|c|c|c|c|}
\hline Year & S/MMBtu & $\frac{\text { Distillate }}{\text { \$MMMBtu }}$ & $\frac{\text { Kerosene }}{\text { S/MMBtu }}$ & $\frac{\mathrm{LPG}^{3}}{\text { S/MMBtu }}$ & $\begin{array}{r}\text { Natural } \\
\text { Gass } \\
\text { \$MMMBtu }\end{array}$ & $\begin{array}{l}\text { Electricity } \\
\text { \$/MMBtu }\end{array}$ \\
\hline $\begin{array}{l}1980 \\
1981 \\
1982 \\
1983 \\
1984\end{array}$ & $\begin{array}{l}4.31 \\
4.93 \\
5.37 \\
5.22 \\
5.13\end{array}$ & $\begin{array}{l}7.05 \\
8.86 \\
8.73 \\
8.12 \\
8.26\end{array}$ & $\begin{array}{l}7.65 \\
9.85 \\
9.69 \\
9.20 \\
9.28\end{array}$ & $\begin{array}{r}9.12 \\
9.85 \\
10.23 \\
10.55 \\
10.03\end{array}$ & $\begin{array}{l}4.85 \\
5.41 \\
6.38 \\
7.67 \\
7.45\end{array}$ & $\begin{array}{l}22.63 \\
28.02 \\
29.37 \\
31.21 \\
32.39\end{array}$ \\
\hline $\begin{array}{l}1985 \\
1986 \\
1987 \\
1988 \\
1989\end{array}$ & $\begin{array}{l}5.82 \\
5.66 \\
5.25 \\
5.46 \\
5.33\end{array}$ & $\begin{array}{l}7.93 \\
6.32 \\
6.35 \\
6.37 \\
6.77\end{array}$ & $\begin{array}{l}8.85 \\
7.65 \\
7.13 \\
7.43 \\
7.59\end{array}$ & $\begin{array}{r}11.12 \\
9.96 \\
9.64 \\
9.53 \\
11.54\end{array}$ & $\begin{array}{l}7.54 \\
7.26 \\
6.68 \\
6.32 \\
7.02\end{array}$ & $\begin{array}{l}32.68 \\
31.74 \\
31.62 \\
31.45 \\
32.47\end{array}$ \\
\hline $\begin{array}{l}1990 \\
1991 \\
1992 \\
1993 \\
1994\end{array}$ & $\begin{array}{l}5.79 \\
5.70 \\
5.77 \\
5.98 \\
5.63\end{array}$ & $\begin{array}{l}8.28 \\
7.87 \\
7.42 \\
7.33 \\
7.20\end{array}$ & $\begin{array}{l}9.76 \\
9.15 \\
8.41 \\
8.35 \\
8.30\end{array}$ & $\begin{array}{l}13.64 \\
14.59 \\
14.43 \\
14.79 \\
14.60\end{array}$ & $\begin{array}{l}7.19 \\
7.16 \\
7.37 \\
7.92 \\
8.49\end{array}$ & $\begin{array}{l}34.32 \\
36.08 \\
37.57 \\
39.71 \\
40.97\end{array}$ \\
\hline
\end{tabular}

\footnotetext{
Anthracite only.

2 Home heating oil.

3 Propane.
} 
New York State Commercial Energy Prices, 1980-1994
Figure 3-2

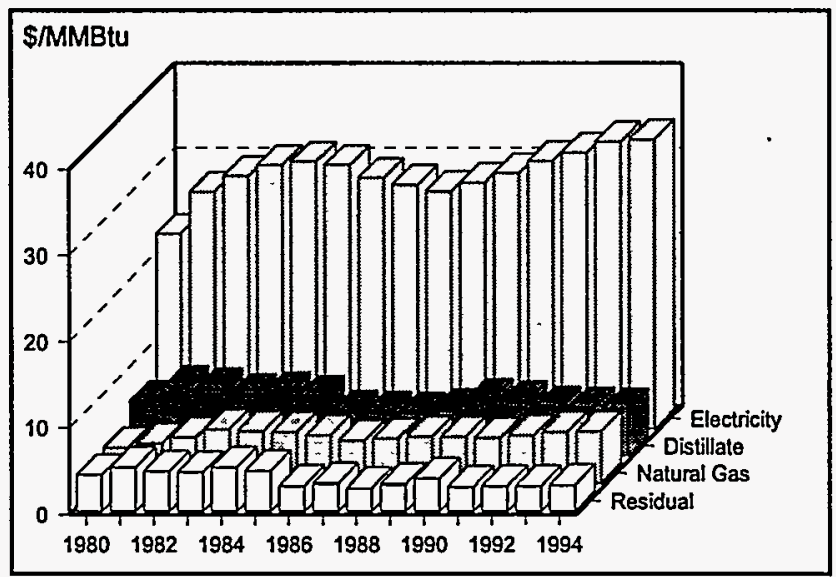

Table 3-2a (Physical Units)

\begin{tabular}{|c|c|c|c|c|c|c|}
\hline Year & $\frac{\text { Coal }}{\text { \$/Ton }}$ & $\begin{aligned} \text { Distillate } \\
\text { Cents/Gal }\end{aligned}$ & $\frac{\text { Residual }}{\text { Cents/Gal }}$ & $\frac{\text { Kerosene }}{\text { Cents/Gal }}$ & $\begin{array}{r}\text { Natural } \\
\text { Gas } \\
\$ \text { \$/Mcf }\end{array}$ & $\frac{\text { Electricity }}{\text { Cents/kWh }}$ \\
\hline $\begin{array}{l}1980 \\
1981 \\
1982 \\
1983 \\
1984\end{array}$ & $\begin{array}{l}37.98 \\
42.68 \\
49.65 \\
48.78 \\
46.19\end{array}$ & $\begin{array}{r}89.83 \\
113.73 \\
110.29 \\
100.68 \\
103.48\end{array}$ & $\begin{array}{l}26.27 \\
31.77 \\
28.92 \\
28.23 \\
31.62\end{array}$ & $\begin{array}{r}92.30 \\
116.87 \\
116.13 \\
102.91 \\
110.41\end{array}$ & $\begin{array}{l}4.27 \\
4.77 \\
5.51 \\
6.51 \\
6.28\end{array}$ & $\begin{array}{r}7.76 \\
9.43 \\
10.06 \\
10.50 \\
10.65\end{array}$ \\
\hline $\begin{array}{l}1985 \\
1986 \\
1987 \\
1988 \\
1989\end{array}$ & $\begin{array}{l}47.62 \\
43.85 \\
44.10 \\
42.76 \\
43.91\end{array}$ & $\begin{array}{l}97.44 \\
74.05 \\
72.70 \\
71.04 \\
76.75\end{array}$ & $\begin{array}{l}29.18 \\
18.39 \\
20.23 \\
16.82 \\
19.61\end{array}$ & $\begin{array}{r}104.00 \\
78.44 \\
77.34 \\
75.70 \\
81.78\end{array}$ & $\begin{array}{l}6.13 \\
5.78 \\
5.17 \\
5.39 \\
5.63\end{array}$ & $\begin{array}{r}10.51 \\
9.99 \\
9.71 \\
9.46 \\
9.79\end{array}$ \\
\hline $\begin{array}{l}1990 \\
1991 \\
1992 \\
1993 \\
1994\end{array}$ & $\begin{array}{l}44.10 \\
43.84 \\
43.98 \\
42.04 \\
42.38\end{array}$ & $\begin{array}{l}92.87 \\
89.03 \\
79.87 \\
78.99 \\
77.54\end{array}$ & $\begin{array}{l}23.59 \\
17.78 \\
18.17 \\
18.22 \\
19.04\end{array}$ & $\begin{array}{l}99.50 \\
94.65 \\
85.25 \\
84.68 \\
77.49\end{array}$ & $\begin{array}{l}5.60 \\
5.49 \\
5.76 \\
6.16 \\
6.23\end{array}$ & $\begin{array}{l}10.18 \\
10.67 \\
11.01 \\
11.45 \\
11.53\end{array}$ \\
\hline
\end{tabular}

Table 3-2b (\$/Million Btu)

\begin{tabular}{|c|r|r|r|r|r|r|}
\hline Year & $\begin{array}{r}\text { Coal } \\
\text { \$MMBtu }\end{array}$ & $\begin{array}{r}\text { Distillate } \\
\text { \$/MMBtu }\end{array}$ & $\begin{array}{r}\text { Residual } \\
\text { \$/MMBtu }\end{array}$ & $\begin{array}{r}\text { Kerosene } \\
\text { \$/MMBtu }\end{array}$ & $\begin{array}{r}\text { Natural } \\
\text { Gas } \\
\text { \$/MMBtu }\end{array}$ & $\begin{array}{r}\text { Electricity } \\
\text { \$/MMBtu }\end{array}$ \\
\hline 1980 & 1.67 & 6.48 & 4.18 & 6.84 & 4.17 & 22.74 \\
1981 & 1.89 & 8.20 & 5.05 & 8.66 & 4.65 & 27.64 \\
1982 & 2.19 & 7.95 & 4.60 & 8.60 & 5.37 & 29.48 \\
1983 & 2.15 & 7.26 & 4.49 & 7.62 & 6.31 & 30.77 \\
1984 & 2.05 & 7.46 & 5.03 & 8.18 & 6.10 & 31.21 \\
\hline 1985 & 2.16 & 7.03 & 4.64 & 7.70 & 5.95 & 30.80 \\
1986 & 1.98 & 5.34 & 2.93 & 5.81 & 5.61 & 29.28 \\
1987 & 1.97 & 5.24 & 3.22 & 5.73 & 5.02 & 28.46 \\
1988 & 1.91 & 5.12 & 2.68 & 5.61 & 5.24 & 27.73 \\
1989 & 1.97 & 5.53 & 3.12 & 6.06 & 5.46 & 28.69 \\
\hline 1990 & 1.96 & 6.70 & 3.75 & 7.37 & 5.43 & 29.84 \\
1991 & 1.95 & 6.42 & 2.83 & 7.01 & 5.33 & 31.27 \\
1992 & 1.98 & 5.76 & 2.89 & 6.31 & 5.59 & 32.27 \\
1993 & 1.90 & 5.70 & 2.90 & 6.27 & 5.99 & 33.56 \\
1994 & 1.92 & 5.59 & 3.03 & 5.74 & 6.06 & 33.79 \\
\hline
\end{tabular}


New York State

Industrial Energy Prices, 1980-1994
Figure 3-3

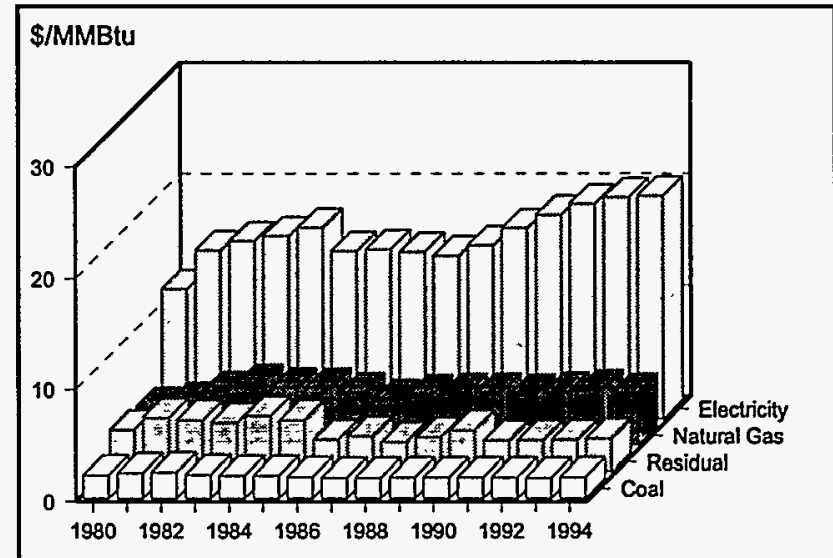

Table 3-3a (Physical Units)

\begin{tabular}{|c|c|c|c|c|c|c|c|}
\hline Year & $\frac{\text { Coal }}{\text { \$Ton }}$ & $\begin{array}{r}\text { Distillate } \\
\text { Cents/Gal }\end{array}$ & $\frac{\text { Residual }}{\$ / \mathrm{bbl}}$ & $\frac{\text { Kerosene }}{\text { Cents/Gal }}$ & $\begin{array}{r}\text { LPG } \\
\text { Cents/Gal }\end{array}$ & $\begin{array}{r}\text { Natural } \\
\text { Gas } \\
\$ / \text { Mcf }\end{array}$ & $\frac{\text { Electricity }}{\text { Cents/kWh }}$ \\
\hline $\begin{array}{l}1980 \\
1981 \\
1982 \\
1983 \\
1984\end{array}$ & $\begin{array}{l}51.37 \\
55.74 \\
57.93 \\
53.06 \\
50.84\end{array}$ & $\begin{array}{l}74.27 \\
91.29 \\
95.47 \\
95.57 \\
89.95\end{array}$ & $\begin{array}{l}23.76 \\
30.33 \\
29.22 \\
27.65 \\
31.62\end{array}$ & $\begin{array}{r}76.73 \\
94.43 \\
101.32 \\
97.81 \\
96.89\end{array}$ & $\begin{array}{l}52.85 \\
55.26 \\
66.50 \\
68.99 \\
73.96\end{array}$ & $\begin{array}{l}3.51 \\
3.99 \\
4.99 \\
5.71 \\
5.37\end{array}$ & $\begin{array}{l}4.00 \\
5.18 \\
5.46 \\
5.62 \\
5.87\end{array}$ \\
\hline $\begin{array}{l}1985 \\
1986 \\
1987 \\
1988 \\
1989\end{array}$ & $\begin{array}{l}50.22 \\
47.54 \\
45.68 \\
45.44 \\
46.13\end{array}$ & $\begin{array}{l}85.07 \\
57.10 \\
60.11 \\
60.52 \\
65.21\end{array}$ & $\begin{array}{l}29.18 \\
18.39 \\
20.23 \\
16.82 \\
19.61\end{array}$ & $\begin{array}{l}91.62 \\
61.49 \\
64.75 \\
65.19 \\
70.24\end{array}$ & $\begin{array}{r}109.13 \\
106.12 \\
103.16 \\
102.41 \\
88.84\end{array}$ & $\begin{array}{l}5.29 \\
4.91 \\
4.28 \\
4.69 \\
4.84\end{array}$ & $\begin{array}{l}5.16 \\
5.20 \\
5.13 \\
5.02 \\
5.34\end{array}$ \\
\hline $\begin{array}{l}1990 \\
1991 \\
1992 \\
1993 \\
1994\end{array}$ & $\begin{array}{l}46.50 \\
46.56 \\
46.91 \\
45.79 \\
45.81\end{array}$ & $\begin{array}{l}86.00 \\
73.12 \\
69.79 \\
67.00 \\
63.65\end{array}$ & $\begin{array}{l}23.59 \\
17.78 \\
18.17 \\
18.22 \\
19.04\end{array}$ & $\begin{array}{l}92.64 \\
78.75 \\
75.17 \\
72.69 \\
63.60\end{array}$ & $\begin{array}{r}94.34 \\
101.26 \\
86.76 \\
88.93 \\
87.78\end{array}$ & $\begin{array}{l}4.86 \\
4.74 \\
4.94 \\
5.17 \\
4.80\end{array}$ & $\begin{array}{l}5.86 \\
6.27 \\
6.62 \\
6.81 \\
6.85\end{array}$ \\
\hline
\end{tabular}

Table 3-3b (\$/Million Btu)

\begin{tabular}{|c|c|c|c|c|c|c|c|}
\hline Year & $\begin{array}{r}\text { Coal } \\
\text { \$/MMBtu }\end{array}$ & $\frac{\text { Distillate }}{\text { S/MMBtu }}$ & $\frac{\text { Residual }}{\text { \$/MMBtu }}$ & $\frac{\text { Kerosene }}{\text { \$MMBtu }}$ & $\begin{array}{r}\text { LPG } \\
\text { S/MMBtu }\end{array}$ & $\begin{array}{r}\text { Natural } \\
\text { Gas } \\
\text { \$MMMBtu }\end{array}$ & $\frac{\text { Electricity }}{\text { \$MMBtu }}$ \\
\hline $\begin{array}{l}1980 \\
1981 \\
1982 \\
1983 \\
1984\end{array}$ & $\begin{array}{l}2.08 \\
2.26 \\
2.34 \\
2.14 \\
2.06\end{array}$ & $\begin{array}{l}5.36 \\
6.58 \\
6.88 \\
6.89 \\
6.49\end{array}$ & $\begin{array}{l}3.78 \\
4.83 \\
4.65 \\
4.40 \\
5.03\end{array}$ & $\begin{array}{l}5.68 \\
7.00 \\
7.51 \\
7.25 \\
7.18\end{array}$ & $\begin{array}{l}5.79 \\
6.05 \\
7.28 \\
7.55 \\
8.10\end{array}$ & $\begin{array}{l}3.43 \\
3.89 \\
4.87 \\
5.54 \\
5.22\end{array}$ & $\begin{array}{l}11.72 \\
15.18 \\
16.00 \\
16.47 \\
17.20\end{array}$ \\
\hline $\begin{array}{l}1985 \\
1986 \\
1987 \\
1988 \\
1989\end{array}$ & $\begin{array}{l}2.06 \\
1.94 \\
1.86 \\
1.85 \\
1.88\end{array}$ & $\begin{array}{l}6.13 \\
4.12 \\
4.33 \\
4.36 \\
4.70\end{array}$ & $\begin{array}{l}4.64 \\
2.93 \\
3.22 \\
2.68 \\
3.12\end{array}$ & $\begin{array}{l}6.79 \\
4.56 \\
4.80 \\
4.83 \\
5.20\end{array}$ & $\begin{array}{r}11.95 \\
11.62 \\
11.30 \\
11.21 \\
9.73\end{array}$ & $\begin{array}{l}5.13 \\
4.78 \\
4.16 \\
4.56 \\
4.69\end{array}$ & $\begin{array}{l}15.12 \\
15.24 \\
15.04 \\
14.71 \\
15.65\end{array}$ \\
\hline $\begin{array}{l}1990 \\
1991 \\
1992 \\
1993 \\
1994\end{array}$ & $\begin{array}{l}1.89 \\
1.89 \\
1.91 \\
1.87 \\
1.88\end{array}$ & $\begin{array}{l}6.20 \\
5.27 \\
5.03 \\
4.83 \\
4.59\end{array}$ & $\begin{array}{l}3.75 \\
2.83 \\
2.89 \\
2.90 \\
3.03\end{array}$ & $\begin{array}{l}6.86 \\
5.83 \\
5.57 \\
5.38 \\
4.71\end{array}$ & $\begin{array}{r}10.33 \\
11.09 \\
9.50 \\
9.74 \\
9.61\end{array}$ & $\begin{array}{l}4.72 \\
4.60 \\
4.79 \\
5.03 \\
4.67\end{array}$ & $\begin{array}{l}17.17 \\
18.38 \\
19.40 \\
19.96 \\
20.08\end{array}$ \\
\hline
\end{tabular}


New York State

Transportation Energy Prices, 1980-1994
Figure 3-4

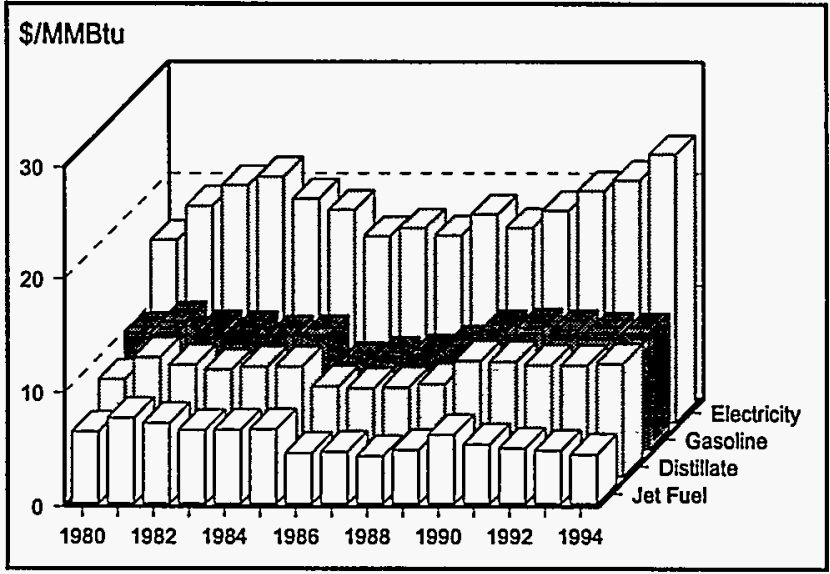

Table 3-4a (Physical Units)

\begin{tabular}{|c|c|c|c|c|c|}
\hline Year & $\frac{\text { Gasoline }}{\text { Cents/Gal }}$ & $\frac{\text { Distillate' }}{\text { Cents/Gal }}$ & $\frac{\text { Jet Fuel }}{\text { Cents/Gal }}$ & $\frac{\text { Residual }^{3}}{\text { Cents/Gal }}$ & $\frac{\text { Electricity }}{\text { Cents/kWh }}$ \\
\hline $\begin{array}{l}1980 \\
1981 \\
1982 \\
1983 \\
1984\end{array}$ & $\begin{array}{l}128.29 \\
140.38 \\
130.71 \\
129.88 \\
124.94\end{array}$ & $\begin{array}{l}118.00 \\
144.80 \\
135.60 \\
130.03 \\
133.59\end{array}$ & $\begin{array}{r}84.69 \\
101.09 \\
94.74 \\
86.94 \\
87.21\end{array}$ & $\begin{array}{l}22.19 \\
27.68 \\
26.26 \\
26.59 \\
28.63\end{array}$ & $\begin{array}{l}5.50 \\
6.54 \\
7.18 \\
7.45 \\
6.77\end{array}$ \\
\hline $\begin{array}{l}1985 \\
1986 \\
1987 \\
1988 \\
1989\end{array}$ & $\begin{array}{r}125.16 \\
95.30 \\
101.54 \\
103.85 \\
114.16\end{array}$ & $\begin{array}{l}133.51 \\
109.66 \\
107.42 \\
108.68 \\
113.05\end{array}$ & $\begin{array}{l}87.90 \\
59.70 \\
61.40 \\
56.00 \\
63.40\end{array}$ & $\begin{array}{l}25.68 \\
14.25 \\
17.87 \\
13.74 \\
16.50\end{array}$ & $\begin{array}{l}6.43 \\
5.62 \\
5.87 \\
5.64 \\
6.28\end{array}$ \\
\hline $\begin{array}{l}1990 \\
1991 \\
1992 \\
1993 \\
1994\end{array}$ & $\begin{array}{l}131.09 \\
133,20 \\
131.46 \\
128.26 \\
127.58\end{array}$ & $\begin{array}{l}140.33 \\
139.73 \\
135.26 \\
134.75 \\
136.81\end{array}$ & $\begin{array}{l}81.41 \\
69.90 \\
65.30 \\
62.95 \\
58.23\end{array}$ & $\begin{array}{l}19.70 \\
14.94 \\
14.69 \\
14.73 \\
15.40\end{array}$ & $\begin{array}{l}5.88 \\
6.40 \\
7.00 \\
7.33 \\
8.13\end{array}$ \\
\hline
\end{tabular}

Table 3-4b (\$/Million Btu)

\begin{tabular}{|c|r|r|r|r|r|}
\hline Year & $\begin{array}{r}\text { Gasoline } \\
\text { \$/MMBtu }\end{array}$ & $\begin{array}{r}\text { Distillate' } \\
\text { \$/MMBtu }\end{array}$ & $\begin{array}{r}\text { Jet Fuel }^{2} \\
\text { \$/MMBtu }\end{array}$ & $\begin{array}{c}\text { Residual }^{3} \\
\text { \$/MMBtu }\end{array}$ & $\begin{array}{c}\text { Electricity }^{4} \\
\text { \$/MMBtu }\end{array}$ \\
\hline 1980 & 10.26 & 8.51 & 6.27 & 3.53 & 16.12 \\
1981 & 11.22 & 10.44 & 7.49 & 4.40 & 19.17 \\
1982 & 10.45 & 9.78 & 7.02 & 4.18 & 21.04 \\
1983 & 10.38 & 9.38 & 6.44 & 4.23 & 21.83 \\
1984 & 9.99 & 9.63 & 6.46 & 4.55 & 19.84 \\
\hline 1985 & 10.01 & 9.63 & 6.51 & 4.09 & 18.85 \\
1986 & 7.62 & 7.91 & 4.42 & 2.27 & 16.47 \\
1987 & 8.12 & 7.75 & 4.55 & 2.84 & 17.20 \\
1988 & 8.30 & 7.84 & 4.15 & 2.19 & 16.53 \\
1989 & 9.13 & 8.15 & 4.70 & 2.63 & 18.41 \\
\hline 1990 & 10.48 & 10.12 & 6.03 & 3.13 & 17.23 \\
1991 & 10.65 & 10.07 & 5.18 & 2.38 & 18.76 \\
1992 & 10.51 & 9.75 & 4.84 & 2.34 & 20.52 \\
1993 & 10.25 & 9.72 & 4.66 & 2.34 & 21.48 \\
1994 & 10.20 & 9.86 & 4.31 & 2.45 & 23.83 \\
\hline
\end{tabular}

\footnotetext{
Diesel.

2 Kerosene-based.

3 Bunker fuel.

4 Consumed by railroads.
} 


\section{Section 4 \\ NEW YORK STATE ENERGY EXPENDITURES}

This section presents the estimated cost of net energy consumption in nominal dollars and in constant 1994 dollars by sector and fuel type for the 1980, 1985, and 1990 to 1994 periods.

Estimated costs of net energy consumption were derived by multiplying quantities of consumption (TBtu) by their respective prices. 
New York State

Energy Expenditure Estimates

by Fuel Type and Sector

in Nominal Dollars

1980-1994
Figure 4-1

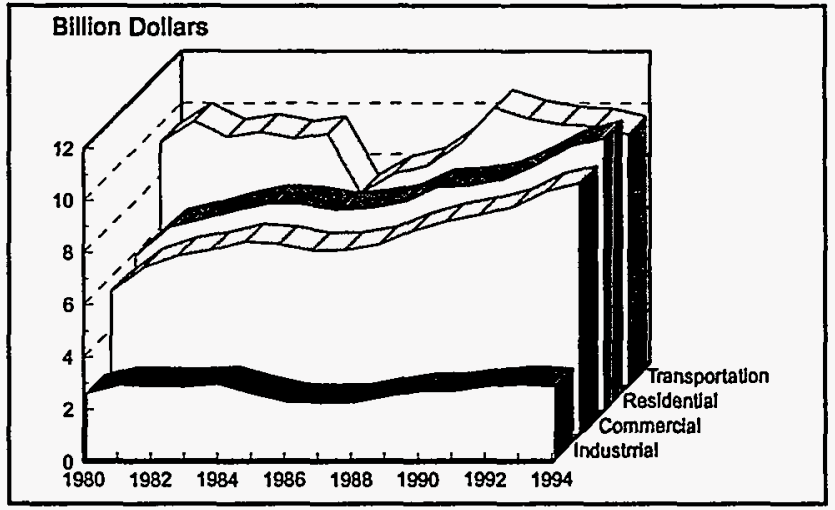

Table 4-1 (Million Dollars)

\begin{tabular}{|c|c|c|c|c|c|c|c|}
\hline & 1980 & 1985 & 1990 & 1991 & 1992 & 1993 & 1994 \\
\hline $\begin{array}{l}\text { RESIDENTIAL } \\
\text { Coal } \\
\text { Petroleum } \\
\quad \text { Distillate } \\
\text { Kerosene } \\
\text { LPG } \\
\text { Natural Gas } \\
\text { Electricity }\end{array}$ & $\begin{array}{r}\$ 17.7 \\
1,782.0 \\
1,618.0 \\
76.5 \\
87.6 \\
1,655.8 \\
2,416.9\end{array}$ & $\begin{array}{r}\$ 27.9 \\
1,656.2 \\
1,444.8 \\
73.5 \\
137.9 \\
2,490.5 \\
3,683.0\end{array}$ & $\begin{array}{r}\$ 16.8 \\
1,563.9 \\
1,286.7 \\
64.4 \\
212.8 \\
2,452.5 \\
4,554.3\end{array}$ & $\begin{array}{r}\$ 17.1 \\
1,524.8 \\
1,169.5 \\
72.3 \\
283.0 \\
2,413.6 \\
4,867.2\end{array}$ & $\begin{array}{r}\$ 19.0 \\
1,551.5 \\
1,217.6 \\
59.7 \\
274.2 \\
2,833.0 \\
4,993.1\end{array}$ & $\begin{array}{r}\$ 16.1 \\
1,549.8 \\
1,231.4 \\
74.3 \\
244.0 \\
3,062.7 \\
5,468.1\end{array}$ & $\begin{array}{r}\$ 11.8 \\
1,418.7 \\
1,128.2 \\
65.6 \\
224.8 \\
3,297.5 \\
5,678.4\end{array}$ \\
\hline Total & $\$ 5,872.4$ & $\$ 7,857.6$ & $\$ 8,587.5$ & $\$ 8,822.7$ & $\$ 9,396.6$ & $\$ 10,096.7$ & $\$ 10,406.4$ \\
\hline $\begin{array}{l}\text { COMMERCIAL } \\
\text { Coal } \\
\text { Petroleum } \\
\text { Distillate } \\
\text { Residual } \\
\text { Kerosene } \\
\text { LPG } \\
\text { Natural Gas } \\
\text { Electricity }\end{array}$ & $\begin{array}{r}\$ 6.5 \\
1,234.1 \\
548.2 \\
669.2 \\
6.8 \\
9.8 \\
612.6 \\
3,597.5\end{array}$ & $\begin{array}{r}\$ 11.2 \\
882.6 \\
487.2 \\
352.2 \\
16.9 \\
26.3 \\
959.7 \\
5,396.2\end{array}$ & $\begin{array}{r}\$ 6.9 \\
925.1 \\
505.2 \\
383.6 \\
7.4 \\
28.9 \\
970.9 \\
6,227.6\end{array}$ & $\begin{array}{r}\$ 6.8 \\
825.1 \\
477.0 \\
304.8 \\
5.6 \\
37.7 \\
963.7 \\
6,579.2\end{array}$ & $\begin{array}{r}\$ 6.5 \\
807.0 \\
466.6 \\
293.6 \\
14.5 \\
32.3 \\
1,142.0 \\
6,741.2\end{array}$ & $\begin{array}{r}\$ 5.1 \\
887.0 \\
501.6 \\
335.2 \\
21.9 \\
28.2 \\
1,230.3 \\
7,181.8\end{array}$ & $\begin{array}{r}\$ 4.6 \\
831.4 \\
474.6 \\
312.1 \\
17.8 \\
26.9 \\
1,441.1 \\
7,332.4\end{array}$ \\
\hline Total & $\$ 5,450.7$ & $\$ 7,249.7$ & $\$ 8,130.5$ & $\$ 8,374.8$ & $\$ 8,696.8$ & $\$ 9,304.3$ & $\$ 9,609.5$ \\
\hline $\begin{array}{l}\text { INDUSTRIAL } \\
\text { Coal } \\
\text { Petroleum } \\
\text { Distillate } \\
\text { Residual } \\
\text { Kerosene } \\
\text { LPG } \\
\text { Natural Gas } \\
\text { Electricity }\end{array}$ & $\begin{array}{r}\$ 304.7 \\
666.4 \\
243.3 \\
352.7 \\
12.5 \\
57.9 \\
414.0 \\
1,109.9\end{array}$ & $\begin{array}{r}\$ 175.3 \\
356.4 \\
168.6 \\
120.6 \\
21.7 \\
45.4 \\
551.5 \\
1,398.6\end{array}$ & $\begin{array}{r}\$ 131.7 \\
258.5 \\
123.4 \\
103.1 \\
6.2 \\
25.8 \\
539.5 \\
1,660.3\end{array}$ & $\begin{array}{r}\$ 128.7 \\
189.9 \\
93.3 \\
42.5 \\
7.6 \\
46.6 \\
554.3 \\
1,720.4\end{array}$ & $\begin{array}{r}\$ 129.3 \\
194.2 \\
91.5 \\
56.6 \\
6.1 \\
39.9 \\
652.4 \\
1,802.3\end{array}$ & $\begin{array}{r}\$ 120.8 \\
231.8 \\
114.0 \\
74.2 \\
7.5 \\
36.0 \\
707.8 \\
1,794.4\end{array}$ & $\begin{array}{r}\$ 120.1 \\
179.2 \\
81.7 \\
61.2 \\
9.4 \\
26.9 \\
670.6 \\
1,785.1\end{array}$ \\
\hline Total & $\$ 2,495.0$ & $\$ 2,481.7$ & $\$ 2,590.1$ & $\$ 2,593,3$ & $\$ 2,778.2$ & $\$ 2,854.8$ & $\$ 2,755.0$ \\
\hline $\begin{array}{l}\text { TRANSPORTATION } \\
\text { Petroleum } \\
\text { Distillate } \\
\text { Residual } \\
\text { Motor Gasoline } \\
\text { Aviation } \\
\text { LPG } \\
\text { Electricity }\end{array}$ & $\begin{array}{r}\$ 9,172.7 \\
497.8 \\
252.4 \\
7,183.0 \\
1,237.7 \\
1.7 \\
129.0\end{array}$ & $\begin{array}{r}\$ 9,459.5 \\
666.4 \\
45.4 \\
7,427.4 \\
1,313.1 \\
7.2 \\
171.5\end{array}$ & $\begin{array}{r}\$ 10,493.3 \\
1,315.6 \\
24.1 \\
7,756.2 \\
1,391.1 \\
6.2 \\
162.0\end{array}$ & $\begin{array}{r}\$ 10,068.1 \\
1,333.3 \\
59.5 \\
7,589.2 \\
1,079.5 \\
6.7 \\
174.5\end{array}$ & $\begin{array}{r}\$ 9,815.2 \\
1,310.4 \\
55.5 \\
7,431.6 \\
1,012.0 \\
5.7 \\
186.7\end{array}$ & $\begin{array}{r}\$ 9,738.8 \\
1,420.1 \\
49.8 \\
7,287.8 \\
976.3 \\
4.9 \\
199.8\end{array}$ & $\begin{array}{r}\$ 9,384.6 \\
1,348.8 \\
49.0 \\
7,071.7 \\
910.3 \\
4.8 \\
235.9\end{array}$ \\
\hline Total & $\$ 9,301.7$ & $\$ 9,631,0$ & $\$ 10,655.2$ & $\$ 10,242.6$ & $\$ 10,002.0$ & $\$ 9,938.6$ & $\$ 9,620,5$ \\
\hline $\begin{array}{l}\text { TOTAL } \\
\text { Coal } \\
\text { Petroleum } \\
\text { Distillate } \\
\text { Residual } \\
\text { Gasoline } \\
\text { Kerosene } \\
\text { Aviation } \\
\text { LPG } \\
\text { Natural Gas } \\
\text { Electricity }\end{array}$ & $\begin{array}{r}\$ 328.9 \\
12,855.2 \\
2,907.4 \\
1,274.3 \\
7,183.0 \\
95.8 \\
1,237.7 \\
157.0 \\
2,682.4 \\
7,253.2\end{array}$ & $\begin{array}{r}\$ 214.5 \\
12,354.6 \\
2,767.0 \\
518.2 \\
7,427.4 \\
112.1 \\
1,313.1 \\
216.8 \\
4,001.7 \\
10,649.3\end{array}$ & $\begin{array}{r}\$ 155.4 \\
13,240.8 \\
3,230.9 \\
510.9 \\
7,756.2 \\
78.0 \\
, 391.1 \\
273.7 \\
3,962.9 \\
12,604.2\end{array}$ & $\begin{array}{r}\$ 152.6 \\
12,607.9 \\
3,073.0 \\
406.7 \\
7,589.2 \\
85.5 \\
1,079.5 \\
374.0 \\
3,931.6 \\
13,341.2\end{array}$ & $\begin{array}{r}\$ 154.9 \\
12,367.9 \\
3,086.1 \\
405.7 \\
7,431.6 \\
80.4 \\
1,012.0 \\
352.1 \\
4,627.5 \\
13,723.2\end{array}$ & $\begin{array}{r}\$ 142.1 \\
12,407.4 \\
3,267.1 \\
459.3 \\
7,287.8 \\
103.8 \\
976.3 \\
313.2 \\
5,000.8 \\
14,644.1\end{array}$ & $\begin{array}{r}\$ 136.6 \\
11,813.9 \\
3,033.4 \\
422.3 \\
7,071.7 \\
92.8 \\
910.3 \\
283.5 \\
5,409.1 \\
15,031.9\end{array}$ \\
\hline Total & $\$ 23,119.7$ & $\$ 27,220.1$ & $\$ 29,963.2$ & $\$ 30,033,4$ & $\$ 30,873.5$ & $\$ 32,194.4$ & $\$ 32,391,5$ \\
\hline
\end{tabular}


New York State

Energy Expenditure Estimates

by Fuel Type and Sector

in Constant ' 94 Dollars

1980-1994
Figure 4-2

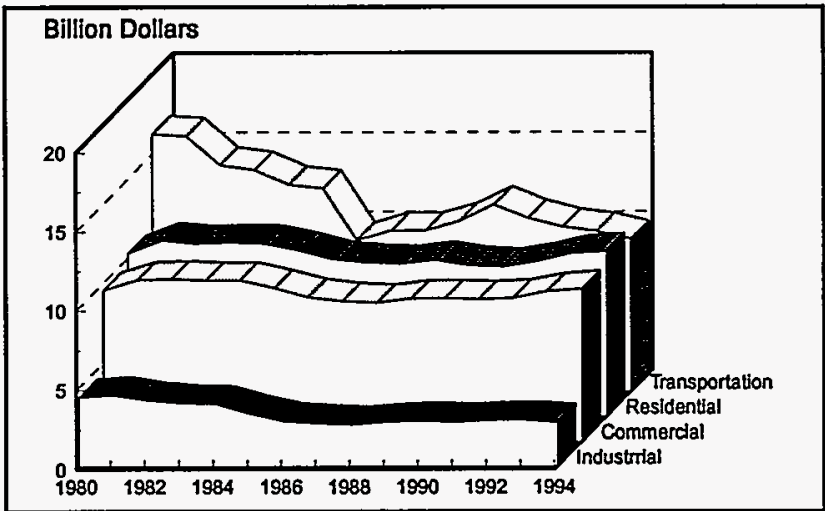

Table 4-2 (Million Dollars)

\begin{tabular}{|c|c|c|c|c|c|c|c|}
\hline & 1980 & 1985 & 1990 & 1991 & 1992 & 1993 & 1994 \\
\hline $\begin{array}{l}\text { RESIDENTIAL } \\
\text { Coal } \\
\text { Petroleum } \\
\text { Distillate } \\
\text { Kerosene } \\
\text { LPG } \\
\text { Natural Gas } \\
\text { Electricity }\end{array}$ & $\begin{array}{r}\$ 30.9 \\
3,120.9 \\
2,833.6 \\
134.0 \\
153.3 \\
2,899.8 \\
4,232.7\end{array}$ & $\begin{array}{r}\$ 37.2 \\
2,208.3 \\
1,926.5 \\
97.9 \\
183.9 \\
3,320.6 \\
4,910.7\end{array}$ & $\begin{array}{r}\$ 18.6 \\
1,735.8 \\
1,428.1 \\
71.5 \\
236.2 \\
2,722.0 \\
5,054.7\end{array}$ & $\begin{array}{r}\$ 18.3 \\
1,630.8 \\
1,250.8 \\
77.3 \\
302.7 \\
2,581.4 \\
5,205.6 \\
\end{array}$ & $\begin{array}{r}\$ 19.8 \\
1,614.5 \\
1,267.0 \\
62.1 \\
285.3 \\
2,948.0 \\
5,195.7 \\
\end{array}$ & $\begin{array}{r}\$ 16.4 \\
1,578.2 \\
1,254.0 \\
75.7 \\
248.5 \\
3,118.8 \\
5,568.3 \\
\end{array}$ & $\begin{array}{r}\$ 11.8 \\
1,418.7 \\
1,128.2 \\
65.6 \\
224.8 \\
3,297.5 \\
5,678.4 \\
\end{array}$ \\
\hline Total & $\$ 10,284.4$ & $\$ 10,476.8$ & $\$ 9,531.0$ & $\$ 9,436.1$ & $\$ 9,778.0$ & $\$ 10,281,7$ & $\$ 10,406.4$ \\
\hline $\begin{array}{l}\text { COMMERCIAL } \\
\text { Coal } \\
\text { Petroleum } \\
\text { Distillate } \\
\text { Residual } \\
\text { Kerosene } \\
\text { LPG } \\
\text { Natural Gas } \\
\text { Electricity }\end{array}$ & $\begin{array}{r}\$ 11.4 \\
2,161.3 \\
960.1 \\
1,172.0 \\
12.0 \\
17.2 \\
1,072.8 \\
6,300.3\end{array}$ & $\begin{array}{r}\$ 15.0 \\
1,176.8 \\
649.6 \\
469.6 \\
22.6 \\
35.1 \\
1,279.6 \\
7,194.9\end{array}$ & $\begin{array}{r}\$ 7.6 \\
1,026.7 \\
560.7 \\
425.8 \\
8.2 \\
32.1 \\
1,077.6 \\
6,911.9\end{array}$ & $\begin{array}{r}\$ 7.3 \\
882.5 \\
510.2 \\
326.0 \\
6.0 \\
40.3 \\
1,030.7 \\
7,036.6\end{array}$ & $\begin{array}{r}\$ 6.8 \\
839.7 \\
485.5 \\
305.5 \\
15.1 \\
33.6 \\
1,188.4 \\
7,014.8\end{array}$ & $\begin{array}{r}\$ 5.2 \\
903.3 \\
510.8 \\
341.4 \\
22.3 \\
28.8 \\
1,252.9 \\
7,313.5\end{array}$ & $\begin{array}{r}\$ 4.6 \\
831.4 \\
474.6 \\
312.1 \\
17.8 \\
26.9 \\
1,441.1 \\
7,332.4\end{array}$ \\
\hline Total & $\$ 9,545,8$ & $\$ 9,666,3$ & $\$ 9,023.8$ & $\$ 8,957.0$ & $\$ 9,049.7$ & $\$ 9,474.9$ & $\$ 9,609.5$ \\
\hline $\begin{array}{l}\text { INDUSTRIAL } \\
\text { Coal } \\
\text { Petroleum } \\
\text { Distillate } \\
\text { Residual } \\
\text { Kerosene } \\
\text { LPG } \\
\text { Natural Gas } \\
\text { Electricity }\end{array}$ & $\begin{array}{r}\$ 533.7 \\
1,167.1 \\
426.2 \\
617.6 \\
21.9 \\
101.4 \\
725.0 \\
1,943.8\end{array}$ & $\begin{array}{r}\$ 233.7 \\
475.1 \\
224.8 \\
160.9 \\
29.0 \\
60.5 \\
735.3 \\
1,864.8\end{array}$ & $\begin{array}{r}\$ 146.2 \\
286.9 \\
136.9 \\
114.5 \\
6.9 \\
28.7 \\
598.8 \\
1,842.8\end{array}$ & $\begin{array}{r}\$ 137.7 \\
203.1 \\
99.8 \\
45.4 \\
8.1 \\
49.8 \\
592.8 \\
1,840.0\end{array}$ & $\begin{array}{r}\$ 134.6 \\
202.1 \\
95.3 \\
58.9 \\
6.4 \\
41.5 \\
678.9 \\
1,875.4\end{array}$ & $\begin{array}{r}\$ 123.0 \\
236.0 \\
116.1 \\
75.6 \\
7.7 \\
36.7 \\
720.7 \\
1,827.3\end{array}$ & $\begin{array}{r}\$ 120.1 \\
179.2 \\
81.7 \\
61.2 \\
9.4 \\
26.9 \\
670.6 \\
1,785.1\end{array}$ \\
\hline Total & $\$ 4,369.6$ & $\$ 3,309.0$ & $\$ 2,874.7$ & $\$ 2,773.5$ & $\$ 2,890.9$ & $\$ 2,907.1$ & $\$ 2,755.0$ \\
\hline $\begin{array}{l}\text { TRANSPORTATION } \\
\text { Petroleum } \\
\text { Distillate } \\
\text { Residual } \\
\text { Motor Gasoline } \\
\text { Aviation } \\
\text { LPG } \\
\text { Electricity }\end{array}$ & $\begin{array}{r}\$ 16,064.3 \\
871.9 \\
442.0 \\
12,579.7 \\
2,167.6 \\
3.0 \\
225.8\end{array}$ & $\begin{array}{r}\$ 12,612.6 \\
888.5 \\
60.5 \\
9,903.2 \\
1,750.8 \\
9.6 \\
228.7\end{array}$ & $\begin{array}{r}\$ 11,646.2 \\
1,460.2 \\
26.7 \\
8,608.5 \\
1,544.0 \\
6.9 \\
179.8 \\
\end{array}$ & $\begin{array}{r}\$ 10,768.0 \\
1,426.0 \\
63.6 \\
8,116.8 \\
1,154.6 \\
7.1 \\
186.6\end{array}$ & $\begin{array}{r}\$ 10,213.6 \\
1,363.6 \\
57.7 \\
7,733.2 \\
1,053.1 \\
5.9 \\
194.3\end{array}$ & $\begin{array}{r}\$ 9,917.3 \\
1,446.1 \\
50.8 \\
7,421.3 \\
994.2 \\
5.0 \\
203.4\end{array}$ & $\begin{array}{r}\$ 9,384.6 \\
1,348.8 \\
49.0 \\
7,071.7 \\
910.3 \\
4.8 \\
235.9\end{array}$ \\
\hline Total & $\$ 16,290.1$ & $\$ 12,841.3$ & $\$ 11,826.0$ & $\$ 10,954.6$ & $\$ 10,407.9$ & $\$ 10,120.8$ & $\$ 9,620.5$ \\
\hline $\begin{array}{l}\text { TOTAL } \\
\text { Coal } \\
\text { Petroleum } \\
\text { Distillate } \\
\text { Residual } \\
\text { Gasoline } \\
\text { Kerosene } \\
\text { Aviation } \\
\text { LPG } \\
\text { Natural Gas } \\
\text { Electricity }\end{array}$ & $\begin{array}{r}\$ 576.0 \\
22,513.6 \\
5,091.7 \\
2,231.7 \\
12,579.7 \\
167.8 \\
2,167.6 \\
275.0 \\
4,697.7 \\
12,702.6 \\
\end{array}$ & $\begin{array}{r}\$ 286.0 \\
16,472.8 \\
3,689.3 \\
691.0 \\
9,903.2 \\
149.5 \\
1,750.8 \\
289.0 \\
5,335.6 \\
14,199.1 \\
\end{array}$ & $\begin{array}{r}\$ 172.5 \\
14,695.7 \\
3,585.9 \\
567.0 \\
8,608.5 \\
86.5 \\
1,544.0 \\
303.8 \\
4,398.3 \\
13,989.1 \\
\end{array}$ & $\begin{array}{r}\$ 163.2 \\
13,484.4 \\
3,286.7 \\
435.0 \\
8,116.8 \\
91.4 \\
1,154.6 \\
400.0 \\
4,204.9 \\
14,268.7 \\
\end{array}$ & $\begin{array}{r}\$ 161.2 \\
12,869.9 \\
3,211.4 \\
422.2 \\
7,733.2 \\
83.6 \\
1,053.1 \\
366.4 \\
4,815.3 \\
14,280.2 \\
\end{array}$ & $\begin{array}{r}\$ 144.7 \\
\$ 12,634.8 \\
\$ 3,327.0 \\
\$ 467.7 \\
\$ 7,421.3 \\
\$ 105.7 \\
\$ 994.2 \\
\$ 318.9 \\
\$ 5,092.4 \\
\$ 14,912.5 \\
\end{array}$ & $\begin{array}{r}\$ 136.6 \\
\$ 11,813.9 \\
\$ 3,033.4 \\
\$ 422.3 \\
\$ 7,071.7 \\
\$ 92.8 \\
\$ 910.3 \\
\$ 283.5 \\
\$ 5,409.1 \\
\$ 15,031.9 \\
\end{array}$ \\
\hline Total & $\$ 40,489.9$ & $\$ 36,293.4$ & $\$ 33,255.5$ & $\$ 32,121.3$ & $\$ 32,126.5$ & $\$ 32,784.5$ & $\$ 32,391.5$ \\
\hline
\end{tabular}




\section{Section 5}

\section{SOURCES OF ENERGY SUPPLIES}

While New York State is the fourth largest energy consumer among all states, only an estimated $7.9 \%$ of total energy requirements are met from resources produced within the State. Hydroelectric power is produced at various locations throughout the State. Oil and natural gas production, primarily located in the western region, amount to $0.1 \%$ and $1.8 \%$ of the State's use of these fuels, respectively.

Households, businesses, industry, and electric utilities in New York State rely on fuels that originate elsewhere in the United States or from abroad. Most of the natural gas used in the State is from Louisiana. Coal resources are from Kentucky, Pennsylvania, Virginia, and West Virginia.

Data on shipments from Petroleum Administration for Defense (PAD) Districts of domestic crude, refined oil, and final destination of imported oil are unavailable for specific state levels. Consequently, levels of oil dependence identified for New York State are estimated by applying PAD District I's dependency to the New York State petroleum-product mix. 
New York State

Primary Energy Production, 1980-1994
Figure 5-1

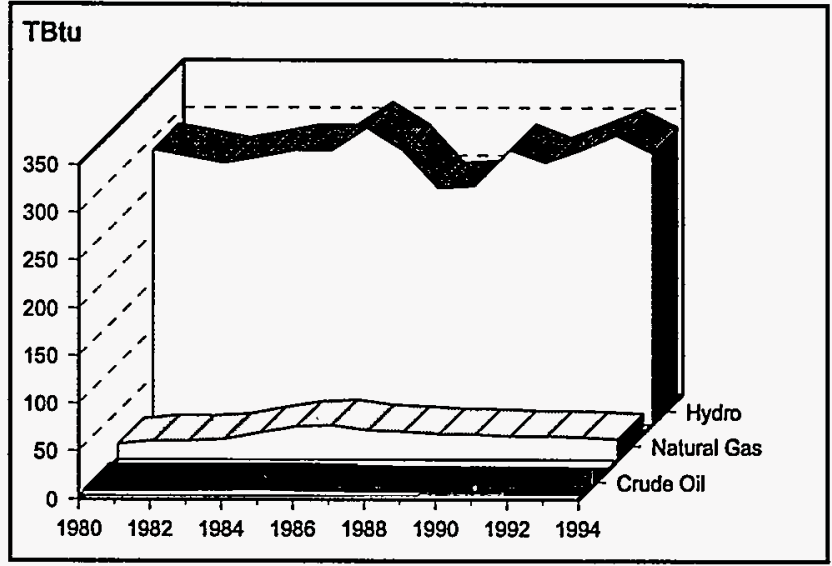

Table 5-1a (Physical Units)

\begin{tabular}{|r|r|r|r|}
\hline Year & $\begin{array}{r}\text { Hydro } \\
\text { Electricity } \\
\text { GWh }\end{array}$ & $\begin{array}{r}\text { Natural } \\
\text { Gas } \\
\text { MMcf }\end{array}$ & $\begin{array}{r}\text { Crude } \\
\text { Qjl } \\
\text { Mbbl }\end{array}$ \\
\hline 1980 & 26,241 & 15,650 & 824 \\
1981 & 25,658 & 19,000 & 849 \\
1982 & 25,329 & 18,760 & 831 \\
1983 & 26,162 & 20,455 & 902 \\
1984 & 26,586 & 27,414 & 952 \\
\hline 1985 & 26,956 & 33,061 & 1071 \\
1986 & 29,480 & 34,796 & 853 \\
1987 & 27,546 & 29,549 & 710 \\
1988 & 23,994 & 28,125 & 567 \\
1989 & 23,918 & 25,673 & 496 \\
\hline 1990 & 27,560 & 25,112 & 417 \\
1991 & 26,257 & 23,438 & 426 \\
1992 & 27,193 & 23,582 & 406 \\
1993 & 28,479 & 22,145 & 341 \\
1994 & 26,800 & 20,476 & 299 \\
\hline
\end{tabular}

Table 5-1b (Trillion Btu)

\begin{tabular}{|c|c|c|c|c|}
\hline Year & $\begin{array}{r}\begin{array}{r}\text { Hydro } \\
\text { Electricity }\end{array} \\
\text { TBtu }\end{array}$ & $\begin{array}{r}\text { Natural } \\
\text { Gas } \\
\text { TBtu }\end{array}$ & $\begin{array}{r}\text { Crude } \\
\text { Qil } \\
\text { TBtu }\end{array}$ & $\frac{\begin{array}{r}\text { Energy } \\
\text { Production }\end{array}}{\text { TBtu }}$ \\
\hline $\begin{array}{l}1980 \\
1981 \\
1982 \\
1983 \\
1984 \\
\end{array}$ & $\begin{array}{l}288.2 \\
280.9 \\
274.0 \\
280.4 \\
287.6\end{array}$ & $\begin{array}{l}16.0 \\
19.5 \\
19.2 \\
21.1 \\
28.2 \\
\end{array}$ & $\begin{array}{l}4.8 \\
4.9 \\
4.8 \\
5.2 \\
5.5\end{array}$ & $\begin{array}{l}309.0 \\
305.3 \\
298.0 \\
306.7 \\
321.4\end{array}$ \\
\hline $\begin{array}{l}1985 \\
1986 \\
1987 \\
1988 \\
1989\end{array}$ & $\begin{array}{l}287.3 \\
311.2 \\
288.2 \\
247.6 \\
249.9\end{array}$ & $\begin{array}{l}34.1 \\
35.8 \\
30.5 \\
28.9 \\
26.5\end{array}$ & $\begin{array}{l}6.2 \\
4.9 \\
4.1 \\
3.3 \\
2.9\end{array}$ & $\begin{array}{l}327.6 \\
352.0 \\
322.8 \\
279.8 \\
279.3\end{array}$ \\
\hline $\begin{array}{l}1990 \\
1991 \\
1992 \\
1993 \\
1994\end{array}$ & $\begin{array}{l}288.0 \\
274.2 \\
287.9 \\
303.6 \\
285.2\end{array}$ & $\begin{array}{l}25.9 \\
24.2 \\
24.3 \\
22.8 \\
21.1\end{array}$ & $\begin{array}{l}2.4 \\
2.5 \\
2.4 \\
2.0 \\
1.7\end{array}$ & $\begin{array}{l}316.3 \\
300.8 \\
314.5 \\
328.4 \\
308.1\end{array}$ \\
\hline
\end{tabular}


New York State and United States Sources of Petroleum Products, 1980-1994
Figure 5-2
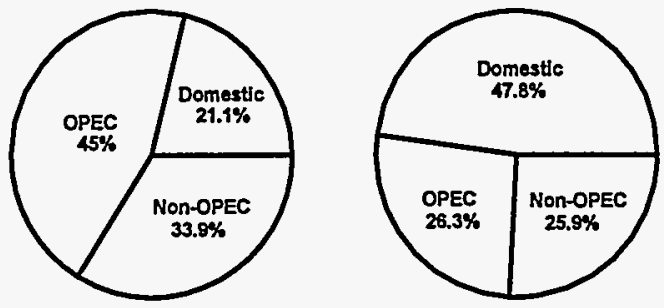

Now York State
United States

Table 5-2a (New York State)

\begin{tabular}{|r|r|r|r|r|}
\hline Year & $\begin{array}{r}\text { Total } \\
\text { Domestic }\end{array}$ & $\begin{array}{r}\text { Total } \\
\text { Foreign }\end{array}$ & OPEC & $\begin{array}{r}\text { Non- } \\
\text { OPEC }\end{array}$ \\
\hline 1980 & $36.0 \%$ & $64.0 \%$ & $47.7 \%$ & $16.3 \%$ \\
1981 & $38.2 \%$ & $61.8 \%$ & $42.3 \%$ & $19.5 \%$ \\
1982 & $43.9 \%$ & $56.1 \%$ & $32.3 \%$ & $23.8 \%$ \\
1983 & $41.6 \%$ & $58.4 \%$ & $29.8 \%$ & $28.6 \%$ \\
1984 & $39.9 \%$ & $60.1 \%$ & $31.0 \%$ & $29.1 \%$ \\
\hline 1985 & $40.7 \%$ & $59.3 \%$ & $27.8 \%$ & $31.5 \%$ \\
1986 & $36.8 \%$ & $63.2 \%$ & $32.5 \%$ & $30.7 \%$ \\
1987 & $32.1 \%$ & $67.9 \%$ & $34.3 \%$ & $33.6 \%$ \\
1988 & $28.7 \%$ & $71.3 \%$ & $37.4 \%$ & $33.9 \%$ \\
1989 & $25.7 \%$ & $74.3 \%$ & $44.3 \%$ & $30.0 \%$ \\
\hline 1990 & $26.1 \%$ & $73.9 \%$ & $47.1 \%$ & $26.8 \%$ \\
1991 & $26.0 \%$ & $74.0 \%$ & $47.5 \%$ & $26.5 \%$ \\
1992 & $25.7 \%$ & $74.3 \%$ & $45.4 \%$ & $28.9 \%$ \\
1993 & $21.8 \%$ & $78.2 \%$ & $46.4 \%$ & $31.8 \%$ \\
1994 & $21.1 \%$ & $78.9 \%$ & $45.0 \%$ & $33.9 \%$ \\
\hline
\end{tabular}

Table 5-2b (United States)

\begin{tabular}{|c|c|c|c|c|}
\hline Year & $\begin{array}{r}\text { Total } \\
\text { Domestic } \\
\end{array}$ & $\begin{array}{r}\text { Total } \\
\text { Eoreign } \\
\end{array}$ & OPEC & $\begin{array}{r}\text { Non- } \\
\text { OPEC } \\
\end{array}$ \\
\hline $\begin{array}{l}1980 \\
1981 \\
1982 \\
1983 \\
1984\end{array}$ & $\begin{array}{r}\text { n.a. } \\
62,7 \% \\
67.8 \% \\
67.4 \% \\
64.5 \%\end{array}$ & $\begin{array}{r}\text { n.a. } \\
37.3 \% \\
32.2 \% \\
32.6 \% \\
35.5 \%\end{array}$ & $\begin{array}{r}\text { n.a. } \\
23.4 \% \\
19.6 \% \\
14.6 \% \\
16.3 \%\end{array}$ & $\begin{array}{r}\text { n.a. } \\
13.9 \% \\
12.6 \% \\
18.0 \% \\
19.2 \%\end{array}$ \\
\hline $\begin{array}{l}1985 \\
1986 \\
1987 \\
1988 \\
1989\end{array}$ & $\begin{array}{l}67.9 \% \\
60.9 \% \\
59.0 \% \\
56.3 \% \\
52.3 \%\end{array}$ & $\begin{array}{l}32.1 \% \\
39.1 \% \\
41.0 \% \\
43.7 \% \\
47.7 \%\end{array}$ & $\begin{array}{l}13.0 \% \\
19.2 \% \\
20.2 \% \\
21.5 \% \\
26.1 \%\end{array}$ & $\begin{array}{l}19.1 \% \\
19.8 \% \\
20.8 \% \\
22.2 \% \\
21.6 \%\end{array}$ \\
\hline $\begin{array}{l}1990 \\
1991 \\
1992 \\
1993 \\
1994\end{array}$ & $\begin{array}{l}51.5 \% \\
53.1 \% \\
52.4 \% \\
48.1 \% \\
47.8 \%\end{array}$ & $\begin{array}{l}48.5 \% \\
46.9 \% \\
47.6 \% \\
51.9 \% \\
52.2 \%\end{array}$ & $\begin{array}{l}27.6 \% \\
26.6 \% \\
26.2 \% \\
27.8 \% \\
26.3 \%\end{array}$ & $\begin{array}{l}20.9 \% \\
20.3 \% \\
21.4 \% \\
24.1 \% \\
25.9 \%\end{array}$ \\
\hline
\end{tabular}


Table A-1 (Heating Degree-Days)

\begin{tabular}{|c|c|c|c|c|c|c|c|c|c|c|c|c|c|}
\hline & Jan. & Feb. & Mar. & Apr. & May & Jun. & Jul. & Aug. & Sep. & Oct. & Nov. & Dec. & Total \\
\hline $\begin{array}{l}1980 \\
1981 \\
1982 \\
1983 \\
1984\end{array}$ & $\begin{array}{l}1127 \\
1381 \\
1384 \\
1097 \\
1264\end{array}$ & $\begin{array}{r}1111 \\
846 \\
998 \\
937 \\
819 \\
\end{array}$ & $\begin{array}{r}915 \\
862 \\
890 \\
782 \\
1045\end{array}$ & $\begin{array}{l}481 \\
436 \\
583 \\
517 \\
528\end{array}$ & $\begin{array}{l}174 \\
197 \\
165 \\
281 \\
286 \\
\end{array}$ & $\begin{array}{l}67 \\
28 \\
73 \\
21 \\
20\end{array}$ & $\begin{array}{l}4 \\
4 \\
4 \\
1 \\
8\end{array}$ & $\begin{array}{r}4 \\
15 \\
32 \\
8 \\
7\end{array}$ & $\begin{array}{r}66 \\
115 \\
97 \\
54 \\
131 \\
\end{array}$ & $\begin{array}{l}428 \\
467 \\
364 \\
350 \\
266 \\
\end{array}$ & $\begin{array}{l}742 \\
661 \\
576 \\
625 \\
657 \\
\end{array}$ & $\begin{array}{r}1160 \\
1034 \\
836 \\
1090 \\
828 \\
\end{array}$ & $\begin{array}{l}6279 \\
6046 \\
6002 \\
5763 \\
5859 \\
\end{array}$ \\
\hline $\begin{array}{l}1985 \\
1986 \\
1987 \\
1988 \\
1989\end{array}$ & $\begin{array}{l}1279 \\
1116 \\
1153 \\
1229 \\
1018\end{array}$ & $\begin{array}{r}979 \\
1052 \\
1056 \\
995 \\
1024\end{array}$ & $\begin{array}{l}780 \\
789 \\
775 \\
843 \\
880\end{array}$ & $\begin{array}{l}434 \\
444 \\
444 \\
531 \\
571\end{array}$ & $\begin{array}{l}172 \\
145 \\
189 \\
198 \\
221\end{array}$ & $\begin{array}{l}70 \\
44 \\
16 \\
43 \\
23\end{array}$ & $\begin{array}{l}7 \\
5 \\
1 \\
1 \\
3\end{array}$ & $\begin{array}{r}16 \\
23 \\
20 \\
5 \\
16\end{array}$ & $\begin{array}{r}62 \\
108 \\
92 \\
113 \\
84\end{array}$ & $\begin{array}{l}328 \\
368 \\
463 \\
507 \\
342\end{array}$ & $\begin{array}{l}580 \\
722 \\
634 \\
621 \\
699\end{array}$ & $\begin{array}{r}1101 \\
939 \\
922 \\
1048 \\
1377\end{array}$ & $\begin{array}{l}5808 \\
5755 \\
5765 \\
6134 \\
6258\end{array}$ \\
\hline $\begin{array}{l}1990 \\
1991 \\
1992 \\
1993 \\
1994\end{array}$ & $\begin{array}{r}895 \\
1115 \\
1079 \\
1037 \\
1388\end{array}$ & $\begin{array}{r}864 \\
856 \\
942 \\
1143 \\
1135\end{array}$ & $\begin{array}{l}779 \\
764 \\
940 \\
966 \\
915\end{array}$ & $\begin{array}{l}479 \\
404 \\
581 \\
501 \\
448\end{array}$ & $\begin{array}{l}281 \\
100 \\
246 \\
185 \\
269\end{array}$ & $\begin{array}{l}29 \\
14 \\
56 \\
36 \\
15\end{array}$ & $\begin{array}{r}4 \\
3 \\
18 \\
2 \\
1\end{array}$ & $\begin{array}{r}12 \\
7 \\
26 \\
8 \\
21\end{array}$ & $\begin{array}{r}97 \\
104 \\
97 \\
106 \\
101\end{array}$ & $\begin{array}{l}260 \\
307 \\
464 \\
427 \\
375\end{array}$ & $\begin{array}{l}590 \\
627 \\
685 \\
675 \\
532 \\
\end{array}$ & $\begin{array}{r}845 \\
924 \\
978 \\
1027 \\
869\end{array}$ & $\begin{array}{l}5135 \\
5225 \\
6112 \\
6113 \\
6069 \\
\end{array}$ \\
\hline Normal & 1158 & 1015 & 870 & 515 & 220 & 32 & 4 & 13 & 88 & 348 & 651 & 1018 & 5932 \\
\hline
\end{tabular}

Table A-2 (Cooling Degree-Days)

\begin{tabular}{|c|c|c|c|c|c|c|c|c|c|c|c|c|c|}
\hline & Jan. & Feb. & Mar. & Apr. & May & Jun. & Jul. & Aug. & Sep. & Oct. & Nov. & Dec. & Total \\
\hline 1980 & 0 & 0 & 0 & 0 & 30 & 70 & 273 & 294 & 93 & 0 & 0 & 0 & 760 \\
1981 & 0 & 0 & 0 & 0 & 22 & 130 & 269 & 193 & 43 & 0 & 0 & 0 & 657 \\
1982 & 0 & 0 & 0 & 0 & 29 & 54 & 244 & 145 & 50 & 0 & 0 & 0 & 522 \\
1983 & 0 & 0 & 0 & 0 & 6 & 139 & 302 & 246 & 105 & 4 & 0 & 0 & 802 \\
1984 & 0 & 0 & 0 & 0 & 10 & 156 & 186 & 252 & 35 & 12 & 0 & 0 & 651 \\
\hline 1985 & 0 & 0 & 0 & 0 & 29 & 59 & 227 & 201 & 87 & 6 & 0 & 0 & 609 \\
1986 & 0 & 0 & 0 & 0 & 40 & 99 & 226 & 158 & 47 & 4 & 0 & 0 & 574 \\
1987 & 0 & 0 & 0 & 0 & 21 & 143 & 283 & 168 & 57 & 0 & 0 & 0 & 672 \\
1988 & 0 & 0 & 0 & 0 & 19 & 99 & 303 & 281 & 43 & 0 & 0 & 0 & 745 \\
1989 & 0 & 0 & 0 & 0 & 15 & 132 & 220 & 195 & 64 & 4 & 0 & 0 & 630 \\
\hline 1990 & 0 & 0 & 0 & 15 & 7 & 110 & 240 & 217 & 54 & 16 & 0 & 0 & 659 \\
1991 & 0 & 0 & 0 & 0 & 71 & 164 & 260 & 258 & 50 & 13 & 0 & 0 & 816 \\
1992 & 0 & 0 & 0 & 0 & 11 & 69 & 163 & 143 & 51 & 0 & 0 & 0 & 437 \\
1993 & 0 & 0 & 0 & 0 & 23 & 113 & 295 & 231 & 51 & 0 & 0 & 0 & 713 \\
1994 & 0 & 0 & 0 & 0 & 7 & 162 & 317 & 153 & 50 & 0 & 0 & 0 & 689 \\
\hline Normal & 0 & 0 & 0 & 0 & 19 & 111 & 244 & 209 & 67 & 5 & 0 & 0 & 655 \\
\hline
\end{tabular}




\section{ABBREVIATIONS}

$\begin{array}{ll}\text { M } & \text { thousand or } 10^{3} \\ \text { MM } & \text { million or } 10^{6} \\ \text { B } & \text { billion or } 10^{9} \\ \text { T } & \text { trillion or } 10^{12} \\ \text { bbl } & \text { barrel } \\ \text { Btu } & \text { British thermal unit } \\ \text { cf } & \text { cubic feet } \\ \text { gal } & \text { gallon } \\ \text { GWh } & \text { gigawatt-hour or million kWh } \\ \text { kWh } & \text { kilowatt-hour } \\ \text { LPG } & \text { liquefied petroleum gas } \\ \text { OPEC } & \text { Organization of Petroleum } \\ & \text { Exporting Countries } \\ \text { N/A } & \text { Not Applicable } \\ \text { n.a. } & \text { Not Available }\end{array}$

\section{CONVERSION FACTORS}

Appropriate heat content of various fuels (1994)

Anthracite coal

Non-utility

Bituminous coal

Electric utility

Residential/commercial

Industrial

Metallurgical

Natural gas

\section{Electric utility}

Non-utility

Hydro

Nuclear

Electricity

Petroleum products

Distillate fuel oil

Jet fuel, kerosene-type

Kerosene

Motor gasoline

LPG (propane)

Residual fuel oil
$16,280,000$ Btu/ton

$\begin{array}{ll}25,916,000 & \text { Btu/ton } \\ 23,004,000 & \text { Btu/ton } \\ 22,036,000 & \text { Btu/ton } \\ 26,800,000 & \text { Btu/ton }\end{array}$

$5,825,000 \quad$ Btu/barrel

$5,670,000 \quad$ Btu/barrel

$5,670,000 \mathrm{Btu} / \mathrm{barrel}$

$5,253,000 \quad \mathrm{Btu} / \mathrm{barr} e l$

$3,836,000 \quad \mathrm{Btu} / \mathrm{barr} e \mathrm{l}$

$6,287,000 \mathrm{Btu} / \mathrm{barrel}$ 


\section{Appendix C Glossary}

\section{GLOSSARY}

anthracite - A hard, black lustrous coal often referred to as "hard coal" that does not burn easily. It has a heating value of 8,500 to 15,000 Btu per pound.

barrel (bbl) - Liquid volume measure equal to 42 gallons, commonly used in expressing quantities of petroleum or petroleum products.

bituminous coal - Often referred to as "soft coal," it is more volatile than anthracite and has a higher heat content than lignite. The most-used coal, it has a heating value of 11,450 to 13,010 Btu per pound.

British thermal unit (Btu) - The quantity of heat necessary to raise the temperature of one pound of water one degree Fahrenheit. Because different energy types use different standards of measurement, they often are converted into Btu to enable comparison. One Btu is equal to 252 calories of heat.

commercial sector - That sector of the state that engages primarily in the sale of services and goods. Included are apartment and office buildings, governmental units, schools, institutions, churches, restaurants, and retail stores.

crude oil - A mixture of hydrocarbons that exist in the liquid phase in natural underground reservoirs. Refined crude oil produces a number of different fuels, including residual fuel, motor gasoline, and distillate.

degree-days, cooling - This statistic is a measure of temperature as it affects energy demand for space cooling. It is similar to heating degree-days, although the relationship is not as precise. If the average of a day's high and low temperature extremes is below 65 degrees $F$, then the cooling degree-days for that day are zero; otherwise, they are equal to the difference between the average and 65 degrees $F$.

degree-days, heating - This statistic is a measure of temperature as it affects energy demand for space heating. It is based on the fact that most buildings require no heat to maintain an inside temperature of at least 70 degrees $F$ when the daily mean is 65 degrees $F$ or higher. If the average of a day's high and low temperature extremes is above 65 degrees $F$, the heating degree-days for that day are taken to be zero; otherwise, they are equal to the different between the average and 65 degrees F. Note that a larger number of heating degree-days implies cooler temperatures.

distillate fuel - Usually means "home heating oil." Its products are actually Nos. 1 and 2 heating oils, diesel fuels, and No. 4 fuel oil. These products are used primarily for space heating, on- and off-highway diesel engine fuel (including railroad engine fuel), and electric power generation.

electric utility - Includes both public and privately owned utilities located within New York State.

end-use - Any ultimate consumption of any type of fossil fuel (petroleum, coal, natural gas) or electricity, whether generated by fossil fuel or other energy sources. End-users are often classified by economic sector, such as residential, commercial, industrial, and transportation. 
feedstock - The raw material furnished to a machine or process. Fossil fuels sometimes are used as feedstocks for their chemical properties, rather than their values as fuel (e.g., oil used to produce plastics and synthetic fabrics).

gallon (gal) - A unit of volume, the U.S. gallon contains 3.785 liters and is .083 times the imperial gallon. One U.S. gallon of water weighs 8.3 pounds.

gigawatt (GW) - 1,000,000 kilowatts, or 1 billion watts.

gigawatthour (GWh) - One billion watthours

hydro - A prefix used to identify a type of generating station, power, or energy output in which the prime mover is water.

industrial sector - That section of the economy involved in either mining, construction, or manufacturing.

jet fuel - Includes both naphtha- and kerosene-type jet fuel that meets the standards for use in aircraft turbine engines. Some jet fuel is used for generating electricity in gas turbines.

kerosene - A petroleum middle distillate with burning properties suitable for use as an illuminant when burned in wick lamps. Kerosene also is used in space heaters, cooking stoves, and water heaters.

kilowatt (kW) - One thousand watts.

kilowatthour (kWh) - The amount of electrical energy involved with a one-kilowatt demand over a period of one hour. One kilowatthour is equivalent to 3,412 Btu of heat energy.

liquefied petroleum gases (LPG) - Propane, propylene, butane, and propane-butane mixtures produced at a refinery or natural gas-processing plant, including plants that fractionate raw natural gas-processing plant liquids. These are derived by refining and processing natural gas, crude oil or unfinished oil.

megawatt (MW) - 1,000 kilowatts or 1 million watts.

megawatthour (MWh) - One million watthours.

motor gasoline - A complex mixture of relatively volatile hydrocarbons, with or without small quantities of additives, that have been blended to form a fuel suitable for use in spark-ignition engines. Included are leaded and unleaded products and refinery products.

natural gas - A mixture of hydrocarbon compounds and small quantities of various nonhydrocarbons existing in the gaseous phase ("gas well" gas) or in solution with crude oil ("oil well" gas) in natural underground reservoirs at reservoir conditions. It comes from the ground with or without accompanying crude oil and is generally much higher in heat content than manufactured gas. It is used as a raw material in the petrochemical industry for manufacturing fertilizer and cellophane.

nuclear - The energy liberated by fission, fusion, or radioactive decay. 
Organization of Petroleum Exporting Countries (OPEC) - OPEC includes Algeria, Ecuador, Gabon, Indonesia, Iran, Iraq, Kuwait, Libya, Nigeria, Qatar, Saudi Arabia, United Arab Emirates, and Venezuela.

petroleum - A generic term applied to oil and oil products in all forms, such as crude oil, lease condensate, unfinished oil, refined nonhydrocarbon compounds blended into finished petroleum products.

propane - A colorless, highly volatile hydrocarbon that is readily recovered as a liquefied gas at natural gas-processing plants and refineries. It is used primarily for residential and commercial heating and cooling, and also as a fuel for transportation and industrial uses, including petrochemical feedstocks. Propane is the first product refined from crude petroleum.

refined petroleum - Products made from processing crude oil, unfinished oils, natural gas liquids and other miscellaneous hydrocarbon compounds. Includes aviation gasoline, motor gasoline, naphtha- and kerosene-type jet fuels, kerosene, distillate fuel oil, residual fuel oil, ethane, liquefied petroleum gases, petrochemical feedstocks, special naphthas, lubricants, paraffin wax, petroleum coke, asphalt, road oil, till gas, and miscellaneous products.

residential sector - Includes private households. Specifically included are the following end-uses: space heating and cooling, water heating, cooking, lighting, clothes drying, and refrigeration.

residual fuel - The heavier oils that remain after the distillate fuel oils and lighter hydrocarbons are boiled off in refinery operations. Included are products knows as No. 5 and No. 6 fuel oil, heavy diesel oil, Navy Special Fuel Oil, Bunker C oil, and acid sludge and pitch used as refinery fuels. Residual fuel oil is used for production of electric power, space heating, vessel bunkering, and various industrial purposes.

trillion $(T)-1,000,000,000,000$, or $10^{12}$.

ton - In the United States, Canada, and Union of South Africa, a unit of weight equal to 2,000 pounds. The American ton is often called the short ton. The metric ton equals 2,204.62 pounds.

watt (W) - The unit of measure for electric power or rate of doing work. The rate of energy transfer equivalent to 1 ampere flowing under a pressure of 1 volt at unity power factor. It is analogous to horsepower or foot-pounds per minute of mechanical power. One horsepower is equivalent to approximately 746 watts.

watthour (Wh) - An electrical energy unit of measure equal to one watt of power supplied to, or taken from an electrical circuit steadily for one hour. 


\section{Appendix D Data Sources}

\section{ANTHRACITE, BITUMINOUS, AND METALLURGICAL COAL}

- Coal Distribution, U.S. Dept. of Energy, Energy Information Administration (U.S.DOE/EIA)

- Quarterly Coal Report (U.S.DOE/EIA)

- State Energy Price and Expenditure Report (U.S.DOE/EIA)

\section{NATURAL GAS}

- Natural Gas Annual (U.S.DOE/EIA)

- New York Gas Report, Long Range Plan (New York Gas Group)

- Central Hudson Gas \& Electric Corp., Annual Reports to the State of New York Public Service Commission (PSC Reports)

- Consolidated Edison Co. of New York, Inc. (PSC Reports)

- Long Island Lighting Company (PSC Reports)

- New York State Electric \& Gas Corp. (PSC Reports)

- Niagara Mohawk Power Corp. (PSC Reports)

- Orange and Rockland Utilities, Inc. (PSC Reports)

- Rochester Gas and Electric Corp. (PSC Reports)

- National Fuel Gas Corporation (PSC Reports)

- Brooklyn Union Gas Company (PSC Reports)

- Corning Natural Gas Corp. (PSC Reports)

- St. Lawrence Gas Company, Inc. (PSC Reports)

- Syracuse Suburban Gas Company, Inc. (PSC Reports)

- Financial Statistics of the Major Privately Owned Utilities in New York State (New York State Dept. of Public Service)

- State Energy Price and Expenditure Report (U.S.DOE/EIA)

\section{CRUDE PETROLEUM AND PETROLEUM PRODUCTS}

- Sales of Fuel Oil and Kerosene (U.S.DOE/EIA)

- Petroleum Supply Annual (U.S.DOE/EIA)

- Petroleum Marketing Monthly (U.S.DOE/EIA)

- Motor Fuel Monthly Gallonage \& Revenue Report (New York State Dept. of Taxation and Finance)

- Monthly Motor Gasoline Reported by States (U.S. Dept. of Transportation, Federal Highway Administration)

- State Energy Price and Expenditure Report (U.S.DOE/EIA) 


\section{ELECTRICITY}

- Report of the Member Systems of the New York Power Pool, Long Range Plan (New York Power Pool)

- Central Hudson Gas \& Electric Corp. (PSC Reports)

- Consolidated Edison Co. of New York, Inc. (PSC Reports)

- Long Island Lighting Company (PSC Reports)

- New York State Electric \& Gas Corp. (PSC Reports)

- Niagara Mohawk Power Corp. (PSC Reports)

- Orange and Rockland Utilities, Inc. (PSC Reports)

- Rochester Gas and Electric Corp. (PSC Reports)

- Annual Reports of the New York Power Authority to the Federal Energy Regulatory Commission

- Power System Statements made by the New York Power Authority to the Energy Information Administration, U.S. Dept. of Energy

- Electric Power Annual (U.S.DOE/EIA)

- Electric Power Quarterly (U.S.DOE/EIA)

- Electric Power Monthly (U.S.DOE/EIA)

- Cost and Quality of Fuels for Electric Utility Plants (U.S.DOE/EIA)

- Statistics of Privately Owned Electric Utilities in the United States (U.S.DOE/EIA)

- Statistics of Publicly Owned Electric Utilities in the United States (U.S.DOE/EIA)

- State Energy Price and Expenditure Report (U.S.DOE/EIA)

\section{UNITED STATES ENERGY DATA}

- Annual Energy Review (U.S.DOE/EIA)

- Monthly Energy Review (U.S.DOE/EIA)

- State Energy Data Review (U.S.DOE/EIA) 